\title{
Cochlear damage affects neurotransmitter chemistry in the central auditory system
}

\author{
Augustine C. Lee $e^{1,2}$ and Donald A. Godfrey ${ }^{1,2}$ * \\ 1 Department of Neurology, University of Toledo College of Medicine, Toledo, OH, USA \\ 2 Division of Otolaryngology and Dentistry, Department of Surgery, University of Toledo College of Medicine, Toledo, OH, USA
}

\author{
Edited by: \\ Jinsheng Zhang, Wayne State \\ University, USA
}

Reviewed by:

Richard Altschuler, University of Michigan, USA

James A. Kaltenbach, Cleveland Clinic, USA

Rui Cai, Southern Illinois University

School of Medicine, USA

${ }^{*}$ Correspondence:

Donald A. Godfrey, Department of Neurology, University of Toledo Health Science Campus, Mail Stop 1195, 3000 Arlington Avenue, Toledo, $\mathrm{OH}$ 43610, USA

e-mail:donald.godfrey@utoledo.edu
Tinnitus, the perception of a monotonous sound not actually present in the environment, affects nearly $20 \%$ of the population of the United States. Although there has been great progress in tinnitus research over the past 25 years, the neurochemical basis of tinnitus is still poorly understood. We review current research about the effects of various types of cochlear damage on the neurotransmitter chemistry in the central auditory system and document evidence that different changes in this chemistry can underlie similar behaviorally measured tinnitus symptoms. Most available data have been obtained from rodents following cochlear damage produced by cochlear ablation, intense sound, or ototoxic drugs. Effects on neurotransmitter systems have been measured as changes in neurotransmitter level, synthesis, release, uptake, and receptors. In this review, magnitudes of changes are presented for neurotransmitter-related amino acids, acetylcholine, and serotonin. A variety of effects have been found in these studies that may be related to animal model, survival time, type and/or magnitude of cochlear damage, or methodology. The overall impression from the evidence presented is that any imbalance of neurotransmitter-related chemistry could disrupt auditory processing in such a way as to produce tinnitus.

Keywords: acetylcholine, aspartate, carboplatin, GABA, glutamate, glycine, taurine, tinnitus

\section{INTRODUCTION}

\section{AN EMERGING ISSUE FOR SOCIETY}

Tinnitus, the perception of a monotonous sound, most commonly ringing (1), not actually present in the environment, can result from many different types of cochlear damage, including especially those resulting from acoustic trauma and ototoxic drugs $(1,2)$. It has been reported that over $10 \%$ of the US population suffers from hearing loss and nearly $20 \%$ from tinnitus specifically (3). Tinnitus and hearing loss were reported as the first and second most prevalent service-connected disabilities of all veterans and together constituted more than a third of the most prevalent disabilities of both all veterans and new veterans (4). Tinnitus has not been a major subject of study until recently. A general PubMed search for "tinnitus" reveals that at least $75 \%$ of all studies related to tinnitus have been published within the last 25 years. A need has been expressed for a better understanding of the rebalancing of excitatory and inhibitory signaling

Abbreviations: AMPA, $\alpha$-amino-3-hydroxy-5-methyl-4-isoxazolepropionic acid; Aud Ctx, auditory cortex; AVCN, anteroventral cochlear nucleus (generally more rostral portion); $\mathrm{CN}$, cochlear nucleus; $\mathrm{CN}$ Granular, cochlear nucleus granular region, usually that portion adjacent to AVCN; CNQX, 5,6-cyano-7-nitro-quinoxaline-2,3dione; DCN, dorsal cochlear nucleus, divided into deep layer and superficial layers (combined fusiform soma and molecular layers); GABA, $\gamma$-aminobutyric acid or $\gamma$ aminobutyrate; IC, inferior colliculus (IC ventral approximately corresponds to the central nucleus of the IC); LSO, lateral superior olivary nucleus; MG, medial geniculate; mRNA, messenger RNA (ribonucleic acid); NMDA, $N$-methyl-D-aspartate; PVCN, posteroventral cochlear nucleus (generally more caudal portion); VCN, ventral cochlear nucleus; VNTB, ventral nucleus of the trapezoid body. mechanisms in auditory disorders (5), but the study of the neurochemical basis for these signaling mechanisms is still in an early stage $(2,5)$.

\section{PURPOSE OF THIS REVIEW}

The vast majority of available data on the neurochemical changes in the central auditory system after cochlear damage is based on animal studies in rodents. These studies have used a variety of post-insult survival times. One study (6) found similar behaviorally measured tinnitus symptoms in chinchillas associated with three different patterns of cochlear damage following acoustic exposure, cisplatin, and carboplatin treatments. In our previous studies, we have found different effects on central auditory system neurotransmitter systems of different types of cochlear damage, including partial damage from acoustic trauma (7-10) and carboplatin treatment $(11,12)$, both of which have been associated with tinnitus $(2,13)$, and complete destruction via cochlear ablation (14-17). Cochlear ablation produces complete transection of auditory nerve fibers, which has been associated with tinnitus (18, 19). Although any chemical change could underlie a hearing disorder (5,20-29), changes in neurotransmitter chemistry would affect the balance of interactions among neurons and could thereby lead to distorted hearing, which might be perceived as tinnitus. The purpose of this review is to compare the effects of various types of cochlear damage on the neurotransmitter chemistry in the central auditory system, thereby to document evidence that different changes in this chemistry can underlie similar behaviorally measured tinnitus symptoms. Although the types of cochlear damage 
employed in these studies can lead to tinnitus, behavioral evidence of tinnitus was not actually assessed in most of the studies.

\section{CATEGORIZATION OF REVIEW COMPONENTS}

Most of the available data are summarized in tables. Effects of intense sound exposure, or acoustic trauma, and ototoxic drugs are compared to those of cochlear ablation. Most available data for ototoxic drugs are for carboplatin, but some data for salicylate, kanamycin, and neomycin are also included. Data from studies that used various survival times are grouped into short (12 weeks), mid (about 1 month), and long ( 2 months or more) times after the event leading to cochlear damage. Although short-term chemical changes could induce other systemic changes related to sustained hearing loss and tinnitus, the chemical changes related to chronic symptoms should presumably be present at long times after cochlear damage. Previous publications have expressed changes as increases, decreases, or no change $(2,5)$. We have taken a more objective approach of presenting the data from various quantitative studies numerically, as percent difference from control. Any change, no matter how small, could theoretically be important, particularly if consistent across neural regions; changes reported to be statistically significant are marked.

\section{NEUROCHEMICALS OF INTEREST}

Most chemical data available for neurotransmitter systems after cochlear damage concern amino acids and acetylcholine. Of the amino acids, glutamate is well established as an excitatory neurotransmitter of auditory nerve fibers (30-35), and there is evidence that it is also a neurotransmitter of ascending (35), interneuronal (36), and descending $(37,38)$ pathways of the auditory system. There is some evidence for aspartate as a neurotransmitter of auditory nerve fibers $(30-32,35)$, but its association with the auditory nerve may also reflect its close metabolic relationship with glutamate $(39,40)$. Glutamine is also closely related metabolically to glutamate as an important precursor $(40,41)$, although predominantly located in glial cells (42). Both glycine and $\gamma$-aminobutyric acid (GABA) are well established as inhibitory neurotransmitters of the central auditory system, especially in the cochlear nucleus (CN), superior olive, and inferior colliculus (IC) $(35,43-55)$. Although taurine is not well established as an inhibitory neurotransmitter, there is evidence that taurine, in addition to its relatively high levels in glia $(42,56)$, is closely associated with GABAergic and glycinergic neurotransmission and may act as an agonist at GABA and glycine receptors (57-59). Available evidence suggests that acetylcholine serves as a neurotransmitter for several centrifugal pathways of the auditory system, particularly olivocochlear and olivo-CN connections $(10,35,36,60,61)$. Its effects are mostly excitatory in the $\mathrm{CN}(62,63)$ as well as other locations (64), and it may function as a neuromodulator as well as a neurotransmitter (64).

Changes in neurotransmitter chemistry have been measured as changes in chemical level; synthetic capacity (synthesis), usually measured as enzyme activity; release, presumably from nerve terminals, by artificial stimulus; tissue uptake rate (uptake); and transmitter receptors (receptors), usually measured by receptor binding or immunohistochemistry. Although data for messenger RNA (mRNA) levels are available for some aspects of neurotransmitter chemistry $(5,52,54)$ and often support the respective protein expression data, we did not include them in the tables of this review because there is often discordance between measurements of mRNA and protein expression (52). This implies that there are other complicating factors that may result in a lack of proportionality between mRNA expression and protein expression.

\section{HISTOLOGICAL EFFECTS OF COCHLEAR DAMAGE}

The most common methods of inducing cochlear damage in animal studies include cochlear ablation, ototoxic drugs, and intense sound (acoustic trauma). The different types of cochlear damage produce distinct histological effects in the central auditory system. Cochlear ablation leads to total degeneration of the auditory

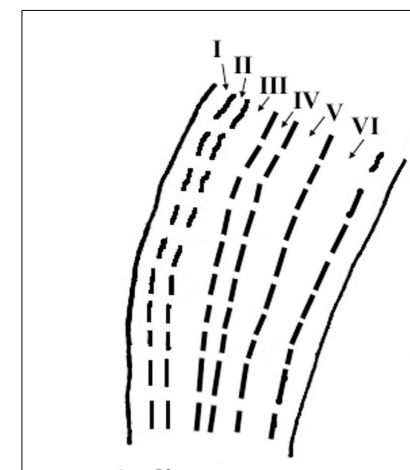

Auditory cortex
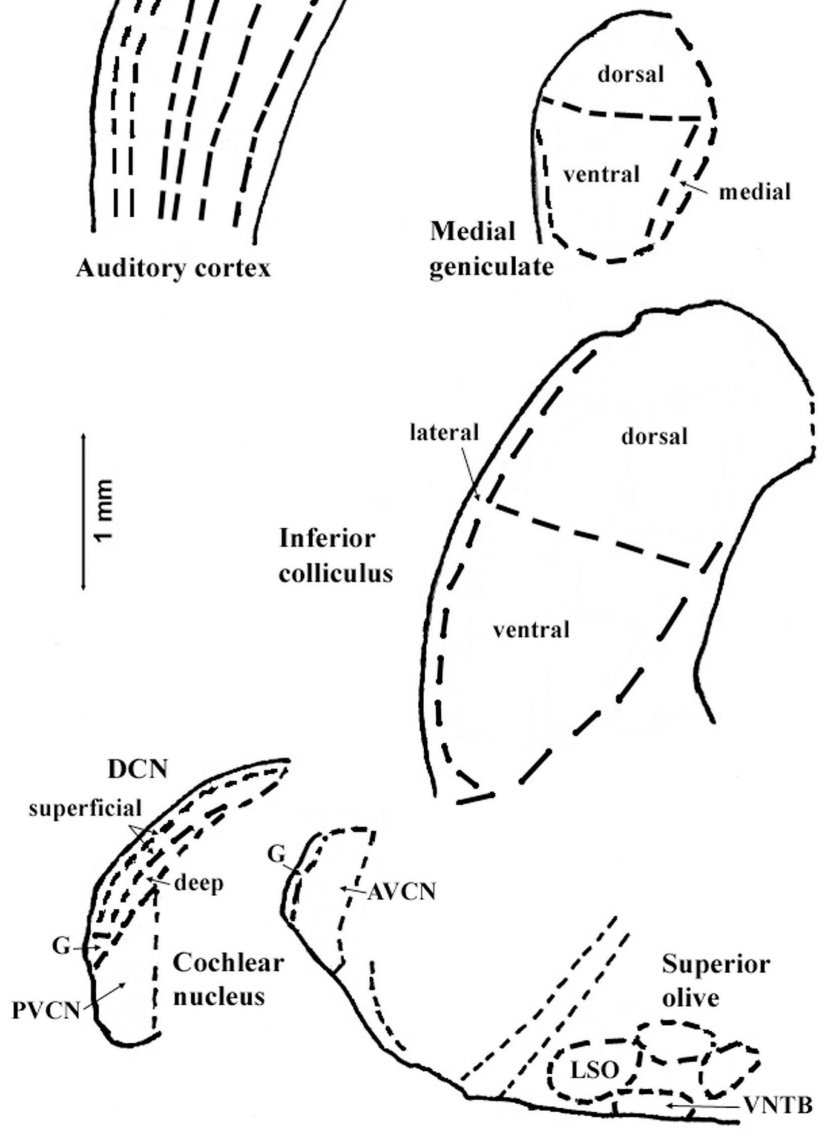

FIGURE 1 | Sections through central auditory regions for which data are shown in the tables. Sections were traced from hamster brain. Names of subregions correspond to those in the tables. Abbreviations: I-VI, layers of auditory cortex; AVCN, anteroventral cochlear nucleus; DCN, dorsal cochlear nucleus; G, granular region; LSO, lateral superior olivary nucleus; PVCN, posteroventral cochlear nucleus; VNTB, ventral nucleus of the trapezoid body. Scale bar is shown at left; dorsal is up, and lateral is left. 
nerve $(65,66)$. Besides degeneration of auditory nerve fibers and terminals in the $\mathrm{CN}$, there is also a hypertrophic reaction of nearby glial cells (67-69) and transneuronal effects in CN neurons and in neurons of higher auditory centers $(66,70)$. Cochlear ablation can also result in delayed, progressive volume decreases in heavily innervated portions of the $\mathrm{CN}(15,16,50)$. The auditory nerve degeneration following acoustic trauma (71-74) or carboplatin (75-77) is only partial. Nevertheless, transneuronal effects of acoustic trauma have been reported (73). Decreases in volume of $\mathrm{CN}$ regions after intense sound have been reported in some studies $(28,74)$ but not others $(9)$. No volume changes in $\mathrm{CN}$ regions were found after carboplatin administration (11).

\section{LIMITATIONS OF CURRENT DATA}

Most data available for effects of cochlear damage on central auditory system chemistry have been obtained in rodents, including guinea pigs, rats, chinchillas, and hamsters. As with any comparison of data among animal species, interpretations are limited by interspecies differences. Another limitation of the data results from differences among individual animals within the same species. For this reason, comparisons between ipsilateral and contralateral sides in the same animal following a unilateral lesion are more reliable than comparisons between individual animals. However, any of the cochlear lesions can have bilateral effects, which can only be detected by comparisons to undamaged control animals.

\section{NOTABLE CHEMICAL CHANGES AFTER COCHLEAR DAMAGE}

The regions represented in the tables are identified in Figure 1. Each table contains available quantitative data for one neurochemical. In the following descriptions, which are keyed to Tables 1-7, we highlight the more prominent chemical changes or patterns presented in the tables. Each item in the tables includes a citation of its respective study.

\section{Box 1 | Table Notes}

Data are presented as percentage changes from control, which was usually an average for control or sham animals, but sometimes (as noted) from the corresponding contralateral structure of animals with unilateral damage. For IC, MG, and Aud Ctx, the affected side is contralateral to the damaged cochlea. Data from the different rodents are marked by $\mathrm{C}$ for chinchilla, $\mathrm{G}$ for guinea pig, $\mathrm{H}$ for hamster, and $\mathrm{R}$ for rat. The terms short, mid, and long refer to survival times after the cochlear damage: at or close to 1 week, about 1 month, and 2 months or more, respectively. Except as noted, data for level and synthesis were from quantitative assays of brain tissue. Data for amino acid levels after carboplatin in chinchilla IC, MG, and Aud Ctx and for acetylcholine synthesis in CN are unpublished data from one of us (DAG; treatment of animals was approved by and in accordance with existing regulations of the University of Toledo Health Science Campus Institutional Animal Care and Use Committee, which are consistent with guidelines of the National Institutes of Health). These data are less reliable since they were obtained from fewer animals, but they give some indication of chemical changes. Average data from mid and long survival times were combined for IC and compared to control chinchilla data.

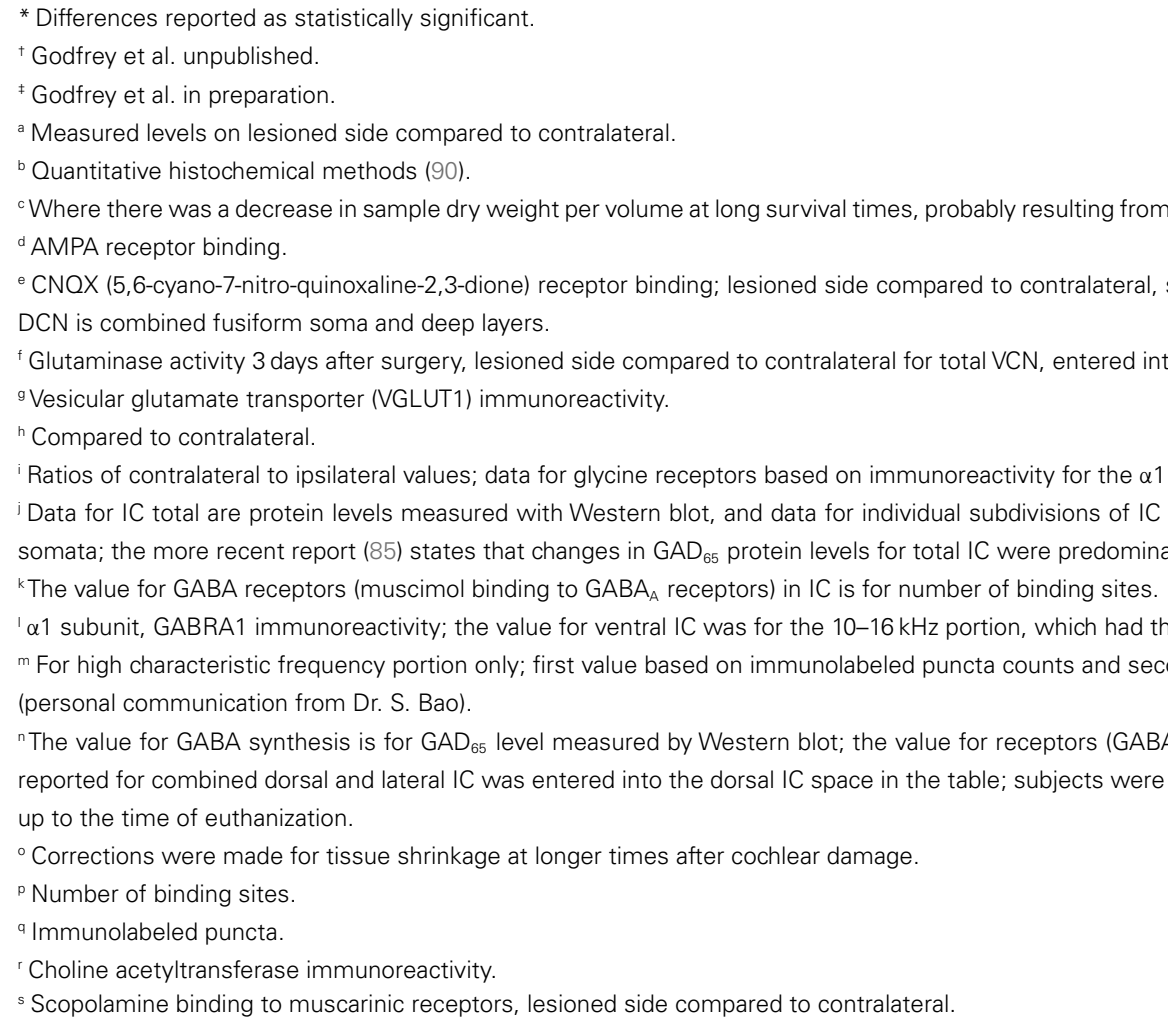


Table 1 | Glutamate.

\begin{tabular}{|c|c|c|c|c|c|c|c|c|c|c|c|}
\hline \multirow[t]{2}{*}{ Region } & \multirow[t]{2}{*}{ Measurement } & \multicolumn{3}{|c|}{ Cochlear ablation } & \multicolumn{3}{|c|}{ Intense sound } & \multicolumn{3}{|c|}{ Carboplatin } & \multirow{2}{*}{$\begin{array}{c}\text { Kanamycin } \\
\text { Short }\end{array}$} \\
\hline & & Short & Mid & Long & Short & Mid & Long & Short & Mid & Long & \\
\hline \multirow[t]{5}{*}{ AVCN } & Level & $\begin{array}{l}-27 G^{*}(31)^{a} \\
-26 R^{*}(17)^{b} \\
-16 C^{*}, \neq, b, c\end{array}$ & $\begin{array}{l}-18 C^{*}, \neq, b, c \\
-13 R(17)^{b}\end{array}$ & $\begin{array}{l}-23 C^{*, \neq, b, c} \\
-8 R(17)^{b}\end{array}$ & & & $+7 \mathrm{H}(9)^{\mathrm{b}}$ & $+5 \mathrm{C}(12)^{\mathrm{b}}$ & $-2 \mathrm{C}(12)^{\mathrm{b}}$ & $\begin{array}{l}-22 \mathrm{C} \\
(12)^{\mathrm{b}}\end{array}$ & \\
\hline & Synthesis & & & & & & & & $-30 C^{*}(11)^{b}$ & & $-63 G *(38)^{9}$ \\
\hline & Release & $-52 \mathrm{G} *(78)$ & $-38 \mathrm{G} *(78)$ & $-19 G(78)$ & $+72 C^{*}(79)^{d}$ & & $+6 C(79)^{d}$ & & & & \\
\hline & Uptake & $-31 G *(78)$ & $-43 G *(78)$ & $-40 G(78)$ & $-60 C^{*}(79)^{d}$ & & $-47 C^{*}(79)^{d}$ & & & & \\
\hline & Receptors & $\begin{array}{l}-35 G^{*}(80)^{d} \\
-25 R^{*}(81)^{e}\end{array}$ & $\begin{array}{l}-15 R(81)^{e} \\
-4 G(80)^{d}\end{array}$ & $+6 G(80)^{d}$ & $0 C(79)^{d}$ & & $+70 C^{*}(79)^{d}$ & & & & \\
\hline \multirow[t]{5}{*}{ PVCN } & Level & $\begin{array}{l}-32 R^{*}(17)^{b} \\
-27 G^{*}(31)^{a} \\
-20 C^{*}(16)^{\ddagger}, b, c\end{array}$ & $\begin{array}{l}-66 C^{*} \\
(16)^{\ddagger, b, c} \\
-36 R^{*}(17)^{b}\end{array}$ & $\begin{array}{l}-59 C^{*} \\
(16)^{\ddagger, b, c} \\
-25 R^{*}(17)^{b}\end{array}$ & $-7 \mathrm{H}(8)^{\mathrm{b}}$ & & $+15 H^{*}(9)^{b}$ & $\mathrm{OC}(12)^{\mathrm{b}}$ & $-6 C(12)^{b}$ & $\begin{array}{l}-39 C^{*} \\
(12)^{b}\end{array}$ & \\
\hline & Synthesis & $-28 G^{*}(33)^{f}$ & & & & & & & $-38 C^{*}(11)^{b}$ & & $-63 G *(38)^{9}$ \\
\hline & Release & $-76 G^{*}(78)$ & $-13 G(78)$ & $-8 G(78)$ & $+131 C^{*}(79)^{d}$ & & $+30 \mathrm{C}(79)^{\mathrm{d}}$ & & & & \\
\hline & Uptake & $-43 G *(78)$ & $-50 G^{*}(78)$ & $-17 \mathrm{G}(78)$ & $-47 C^{*}(79)^{d}$ & & $-50 C^{*}(79)^{d}$ & & & & \\
\hline & Receptors & $\begin{array}{l}-14 R^{*}(81)^{e} \\
+83 G^{*}(80)^{d}\end{array}$ & $\begin{array}{l}-14 R(81)^{e} \\
-10 G(80)^{d}\end{array}$ & $-13 G(80)^{d}$ & $-5 C(79)^{d}$ & & $+62 C^{*}(79)^{d}$ & & & & \\
\hline \multirow[t]{3}{*}{ DCN total } & Synthesis & $-2 \mathrm{G}(33)^{f}$ & & & & & & & & & \\
\hline & Release & $-50 G *(78)$ & $-53 G *(78)$ & $-52 \mathrm{G} *(78)$ & $+72 C^{*}(79)^{d}$ & & $+44 \mathrm{C}(79)^{\mathrm{d}}$ & & & & \\
\hline & Uptake & $-38 G *(78)$ & $-25 G(78)$ & $+21 \mathrm{G}(78)$ & $-41 C^{*}(79)^{d}$ & & $-12 C(79)^{d}$ & & & & \\
\hline \multirow[t]{3}{*}{ DCN deep } & Level & $\begin{array}{l}-19 R(17)^{b}, \\
-18 C^{\ddagger, b, c},-6 G \\
(31)^{a}\end{array}$ & $\begin{array}{l}-22 C^{\ddagger, b, c} \\
-15 R(17)^{b}\end{array}$ & $\begin{array}{l}-21 C^{\ddagger, b, c} \\
-14 R(17)^{b}\end{array}$ & $-16 H^{*}(8)^{b}$ & $-9 \mathrm{H}(8)^{\mathrm{b}}$ & $+16 \mathrm{H}(9)^{\mathrm{b}}$ & $+8 \mathrm{C}(12)^{\mathrm{b}}$ & $-1 \mathrm{C}(12)^{\mathrm{b}}$ & $\begin{array}{l}-6 C \\
(12)^{b}\end{array}$ & \\
\hline & Synthesis & & & & & & & & $-5 C(11)^{b}$ & & $+13 \mathrm{G}(38)^{\mathrm{g}}$ \\
\hline & Receptors & $\begin{array}{l}-18 R^{*}(81)^{e} \\
+60 G^{*}(80)^{d}\end{array}$ & $\begin{array}{l}-17 R(81)^{e} \\
0 G(80)^{d}\end{array}$ & $+5 G(80)^{d}$ & & & & & & & \\
\hline \multirow[t]{4}{*}{ DCN superficial } & Level & $\begin{array}{l}-10 C^{\ddagger, b, c},-6 R \\
(17)^{b},+7 G(31)^{a}\end{array}$ & $\begin{array}{l}-9 R(17)^{b} \\
-2 C^{\ddagger}, b, c\end{array}$ & $\begin{array}{l}-8 C^{\ddagger, b, c} \\
-3 R(17)^{b}\end{array}$ & $-13 H^{*}(8)^{b}$ & $-3 \mathrm{H}(8)^{\mathrm{b}}$ & $+6 \mathrm{H}(9)^{\mathrm{b}}$ & $+3 \mathrm{C}(12)^{\mathrm{b}}$ & $-5 C(12)^{b}$ & $\mathrm{OC}(12)^{\mathrm{b}}$ & \\
\hline & Synthesis & & & & & & & & $-8 \mathrm{C}(11)^{\mathrm{b}}$ & & OG $(38)^{\mathrm{g}}$ \\
\hline & Receptors & $+8 \mathrm{R}(81)^{\mathrm{e}}$ & $-7 G(80)^{d}$ & $-5 G(80)^{d}$ & OC $(79)^{d}$ & & $-10 C(79)^{d}$ & & & & \\
\hline & & $+10 \mathrm{G}(80)^{\mathrm{d}}$ & $+3 \mathrm{R}(81)^{\mathrm{e}}$ & & & & & & & & \\
\hline \multirow[t]{3}{*}{$\mathrm{CN}$ granular } & Level & $\begin{array}{l}-18 R(17)^{b} \\
-16 G(31)^{a} \\
-14 C^{\ddagger, b, c}\end{array}$ & $\begin{array}{l}-3 R(17)^{b} \\
+8 C^{\ddagger, b, c}\end{array}$ & $\begin{array}{l}-12 R(17)^{b}, \\
O C^{\ddagger}, b, c\end{array}$ & & & $+5 \mathrm{H}(9)^{b}$ & & $-7 \mathrm{C}(11)^{\mathrm{b}}$ & & \\
\hline & Synthesis & & & & & & & & $-8 \mathrm{C}(11)^{\mathrm{b}}$ & & $+7 \mathrm{G}(38)^{\mathrm{g}}$ \\
\hline & Receptors & $-2 \mathrm{G}(80)^{\mathrm{d}}$ & $-24 G^{*}(80)^{d}$ & $+9 G(80)^{d}$ & & & & & & & \\
\hline \multirow[t]{4}{*}{ LSO } & Level & $+16 \mathrm{R}(17)^{\mathrm{b}}$ & $+10 \mathrm{R}(17)^{\mathrm{b}}$ & $-24 R(17)^{b}$ & & & & & & & \\
\hline & Release & $-20 \mathrm{G}(78)$ & $+1 \mathrm{G}(78)$ & $+18 \mathrm{G} *(78)$ & & & & & & & \\
\hline & Uptake & $-18 G *(78)$ & $-1 \mathrm{G}(78)$ & $+34 G^{*}(78)$ & & & & & & & \\
\hline & Receptors & $-39 G *(80)^{d}$ & $-9 \mathrm{G}(80)^{\mathrm{d}}$ & $-23 G *(80)^{d}$ & & & & & & & \\
\hline \multirow[t]{2}{*}{ IC dorsal } & Level & & & & & & $+12 \mathrm{H}^{*}(9)^{\mathrm{b}}$ & & $\begin{array}{l}-3 c \\
\left(31^{a}, 78\right)^{\dagger, b}\end{array}$ & & \\
\hline & Receptors & $+6 G(80)^{d}$ & $-4 G(80)^{d}$ & $+2 \mathrm{G}(80)^{\mathrm{d}}$ & & & & & & & \\
\hline \multirow[t]{4}{*}{ IC ventral } & Level & & & & & & $+10 H^{*}(9)^{b}$ & & $+15 C^{\dagger, b}$ & & \\
\hline & Release & $+14 \mathrm{G}(78)$ & $+55 \mathrm{G} *(78)$ & $+41 \mathrm{G}^{*}(78)$ & & & & & & & \\
\hline & Uptake & $-14 \mathrm{G}(78)$ & $-5 \mathrm{G}(78)$ & $-5 G(78)$ & & & & & & & \\
\hline & Receptors & $+12 \mathrm{G}(80)^{\mathrm{d}}$ & $+18 G(80)^{d}$ & $+6 \mathrm{G}(80)^{\mathrm{d}}$ & & & & & & & \\
\hline \multirow[t]{2}{*}{ IC lateral } & Level & & & & & & $+7 \mathrm{H}(9)^{\mathrm{b}}$ & & $+16 C^{t, b}$ & & \\
\hline & Receptors & $+16 \mathrm{G}(80)^{d}$ & $-2 G(80)^{d}$ & $-9 G^{*}(80)^{d}$ & & & & & & & \\
\hline MG total & Level & & & & & & & $+7 C^{\dagger, b}$ & $+7 C^{\dagger, b}$ & $+1 C^{t, b}$ & \\
\hline MG dorsal & Level & & & & & & $+6 \mathrm{H}(9)^{b}$ & & & & \\
\hline MG ventral & Level & & & & & & $+2 \mathrm{H}(9)^{\mathrm{b}}$ & & & & \\
\hline MG medial & Level & & & & & & $-2 \mathrm{H}(9)^{\mathrm{b}}$ & & & & \\
\hline
\end{tabular}


Table 1 | Continued

\begin{tabular}{|c|c|c|c|c|c|c|c|c|c|c|c|}
\hline \multirow[t]{2}{*}{ Region } & \multirow[t]{2}{*}{ Measurement } & \multicolumn{3}{|c|}{ Cochlear ablation } & \multicolumn{3}{|c|}{ Intense sound } & \multicolumn{3}{|c|}{ Carboplatin } & \multirow{2}{*}{$\begin{array}{c}\text { Kanamycin } \\
\text { Short }\end{array}$} \\
\hline & & Short & Mid & Long & Short & Mid & Long & Short & Mid & Long & \\
\hline Aud Ctx total & Level & & & & & & & $+5 C^{\dagger, b}$ & $+4 C^{\dagger, b}$ & $-8 C^{\dagger, b}$ & \\
\hline Aud Ctx layer I & Level & & & & & & $+6 \mathrm{H}(9)^{b}$ & & & & \\
\hline Aud Ctx layer II & Level & & & & & & $+6 \mathrm{H}(9)^{\mathrm{b}}$ & & & & \\
\hline Aud Ctx layer III & Level & & & & & & $+6 \mathrm{H}(9)^{\mathrm{b}}$ & & & & \\
\hline Aud Ctx layer IV & Level & & & & & & $+8 \mathrm{H}(9)^{\mathrm{b}}$ & & & & \\
\hline Aud Ctx layer V & Level & & & & & & $+8 \mathrm{H}(9)^{b}$ & & & & \\
\hline Aud Ctx layer VI & Level & & & & & & $+8 \mathrm{H}(9)^{\mathrm{b}}$ & & & & \\
\hline
\end{tabular}

See Box 1 for table notes.

Table 2 | Aspartate.

\begin{tabular}{|c|c|c|c|c|c|c|c|c|c|c|}
\hline \multirow[t]{2}{*}{ Region } & \multirow[t]{2}{*}{ Measurement } & \multicolumn{3}{|c|}{ Cochlear ablation } & \multicolumn{3}{|c|}{ Intense sound } & \multicolumn{3}{|c|}{ Carboplatin } \\
\hline & & Short & Middle & Long & Short & Mid & Long & Short & Mid & Long \\
\hline AVCN & Level & $\begin{array}{l}-42 G^{*}(31)^{a},-19 R^{*} \\
(17)^{b},+13 C^{\ddagger, b, c}\end{array}$ & $\begin{array}{l}-19 C^{*} \neq, \mathrm{b}, \mathrm{c} \\
\mathrm{OR}(17)^{\mathrm{b}}\end{array}$ & $\begin{array}{l}-18 R(17)^{b} \\
-12 C^{\ddagger, b, c}\end{array}$ & & & $+17 H^{*}(9)^{b}$ & $-20 \mathrm{C}(12)^{b}$ & $-27 C^{*}(12)^{b}$ & $-28 C^{*}(12)^{b}$ \\
\hline PVCN & Level & $\begin{array}{l}-37 G^{*}(31)^{a},-31 R^{*} \\
(17)^{b},+58 C^{*}, \neq, b, c\end{array}$ & $\begin{array}{l}-63 C^{*} \neq, b, c \\
-31 R^{*}(17)^{b}\end{array}$ & $\begin{array}{l}-57 C^{*, \neq, b, c} \\
-18 R(17)^{b}\end{array}$ & $-11 \mathrm{H}(8)^{\mathrm{b}}$ & & $+36^{*} \mathrm{H}(9)^{\mathrm{b}}$ & $-14 \mathrm{C}(12)^{\mathrm{b}}$ & $-24 C(12)^{b}$ & $-40 C^{*}(12)^{b}$ \\
\hline DCN deep & Level & $\begin{array}{l}-16 \mathrm{R}(17)^{\mathrm{b}},-7 \mathrm{G} \\
(31)^{\mathrm{a}},+19 \mathrm{C}^{\ddagger}, \mathrm{b}, \mathrm{c}\end{array}$ & $\begin{array}{l}-30 C^{\ddagger}, b, c \\
+3 R(17)^{b}\end{array}$ & $\begin{array}{l}-24 C^{\ddagger, b, c},-4 R \\
(17)^{b}\end{array}$ & $-3 \mathrm{H}(8)^{\mathrm{b}}$ & $-30 \mathrm{H}(8)^{\mathrm{b}}$ & $+21 \mathrm{H}(9)^{\mathrm{b}}$ & $-19 \mathrm{C}(12)^{\mathrm{b}}$ & $-9 \mathrm{C}(12)^{\mathrm{b}}$ & $-19 C^{*}(12)^{b}$ \\
\hline DCN superficial & Level & $\begin{array}{l}-9 C^{\ddagger}, b, c,-5 R(17)^{b}, \\
O G(31)^{a}\end{array}$ & $\begin{array}{l}-8 C^{\ddagger, b, c},-3 R \\
(17)^{b}\end{array}$ & $\begin{array}{l}-1 R(17)^{b} \\
+2 C^{\ddagger}, b, c\end{array}$ & $-8 \mathrm{H}(8)^{b}$ & $-13 \mathrm{H}(8)^{b}$ & $+11 \mathrm{H}(9)^{\mathrm{b}}$ & $-6 C(12)^{b}$ & $-2 \mathrm{C}(12)^{\mathrm{b}}$ & $-10 \mathrm{C}(12)^{\mathrm{b}}$ \\
\hline $\mathrm{CN}$ granular & Level & $\begin{array}{l}-10 G(31)^{a},-7 C^{\ddagger, b, c} \\
+2 R(17)^{b}\end{array}$ & $\begin{array}{l}-6 C^{\ddagger, b, c},+9 R \\
(17)^{b}\end{array}$ & $\begin{array}{l}-13 R(17)^{b} \\
-2 C^{\ddagger}, b, c\end{array}$ & & & $+23 \mathrm{H}^{*}(9)^{\mathrm{b}}$ & & $-4 C(11)^{b}$ & \\
\hline LSO & Level & $+18 \mathrm{R}(17)^{\mathrm{b}}$ & $+4 \mathrm{R}(17)^{\mathrm{b}}$ & $-39 R(17)^{b}$ & & & & & & \\
\hline IC dorsal & Level & & & & & & $+12 \mathrm{H}^{*}(9)^{\mathrm{b}}$ & & $+9 c^{\dagger, b}$ & \\
\hline IC ventral & Level & & & & & & $+10 H^{*}(9)^{b}$ & & $-1 C^{\dagger, b}$ & \\
\hline IC lateral & Level & & & & & & $+12 \mathrm{H}^{*}(9)^{b}$ & & $+31 C^{\dagger, b}$ & \\
\hline MG total & Level & & & & & & & $-16 C^{\dagger, b}$ & $-14 C^{\dagger, b}$ & $-22 C^{\dagger, b}$ \\
\hline MG dorsal & Level & & & & & & $+13 \mathrm{H}^{*}(9)^{\mathrm{b}}$ & & & \\
\hline MG ventral & Level & & & & & & $+12 \mathrm{H}^{*}(9)^{\mathrm{b}}$ & & & \\
\hline MG medial & Level & & & & & & $+12 \mathrm{H}^{*}(9)^{b}$ & & & \\
\hline Aud Ctx total & Level & & & & & & & $-7 C^{\dagger, b}$ & $+2 C^{\dagger, b}$ & $-25 C^{*}, t, b$ \\
\hline Aud Ctx layer I & Level & & & & & & $+12 \mathrm{H}(9)^{\mathrm{b}}$ & & & \\
\hline Aud Ctx layer II & Level & & & & & & $+13 \mathrm{H}(9)^{\mathrm{b}}$ & & & \\
\hline Aud Ctx layer III & Level & & & & & & $+10 \mathrm{H}(9)^{\mathrm{b}}$ & & & \\
\hline Aud Ctx layer IV & Level & & & & & & $+7 \mathrm{H}(9)^{\mathrm{b}}$ & & & \\
\hline Aud Ctx layer V & Level & & & & & & $+16 H^{*}(9)^{b}$ & & & \\
\hline Aud Ctx layer VI & Level & & & & & & $+21 \mathrm{H}^{*}(9)^{\mathrm{b}}$ & & & \\
\hline
\end{tabular}

See Box 1 for table notes.

\section{GLUTAMATE}

In all three species studied, cochlear ablation resulted in decreased glutamate levels in each time category and in all regions receiving sizable innervation from auditory nerve fibers (AVCN, PVCN, and deep DCN, Table 1). Effects in superficial DCN and granular regions were smaller and inconsistent. Glutamate release was also consistently decreased at all times. Uptake was decreased in each time category of the $\mathrm{CN}$ regions except in the DCN at long survival times. Changes in glutamate receptors (AMPA type) were not consistent in guinea pig, but in rat they were all 
Table 3 | Glutamine.

\begin{tabular}{|c|c|c|c|c|c|c|c|c|c|c|}
\hline \multirow[t]{2}{*}{ Region } & \multirow[t]{2}{*}{ Measurement } & \multicolumn{3}{|c|}{ Cochlear ablation } & \multicolumn{3}{|c|}{ Intense sound } & \multicolumn{3}{|c|}{ Carboplatin } \\
\hline & & Short & Mid & Long & Short & Mid & Long & Short & Mid & Long \\
\hline AVCN & Level & $\begin{array}{l}-9 C^{*}, \neq, b, c \\
-6 R(17)^{b}\end{array}$ & $\begin{array}{l}+4 C^{\ddagger}, b, c \\
+6 R(17)^{b}\end{array}$ & $\begin{array}{l}+12 \mathrm{R}(17)^{\mathrm{b}} \\
+16 \mathrm{C}^{*}, \neq, \mathrm{b}, \mathrm{c}\end{array}$ & & & $-2 \mathrm{H}(9)^{\mathrm{b}}$ & $+6 \mathrm{C}(12)^{\mathrm{b}}$ & $+14 \mathrm{C}(12)^{b}$ & $+16 \mathrm{C}(12)^{\mathrm{b}}$ \\
\hline PVCN & Level & $\begin{array}{l}-7 C^{\ddagger, b, c} \\
+2 R(17)^{b}\end{array}$ & $\begin{array}{l}-22 C^{*} \neq, b, c \\
+11 R(17)^{b}\end{array}$ & $\begin{array}{l}+1 C^{\ddagger}, b, c \\
+35 R^{*}(17)^{b}\end{array}$ & $-3 H(8)^{b}$ & & $+3 \mathrm{H}(9)^{\mathrm{b}}$ & $+17 \mathrm{C}(12)^{b}$ & $+11 \mathrm{C}(12)^{\mathrm{b}}$ & $+3 \mathrm{C}(12)^{\mathrm{b}}$ \\
\hline DCN deep & Level & $\begin{array}{l}-19 C^{*,} \neq, b, c \\
+2 R(17)^{b}\end{array}$ & $\begin{array}{l}-8 C^{\ddagger, b, c} \\
+16 R^{*}(17)^{b}\end{array}$ & $\begin{array}{l}-12 C^{\ddagger, b, c} \\
+21 R^{*}(17)^{b}\end{array}$ & $+11 \mathrm{H}(8)^{\mathrm{b}}$ & $-23 \mathrm{H}(8)^{\mathrm{b}}$ & $\mathrm{OH}(9)^{b}$ & $+19 \mathrm{C}(12)^{b}$ & $+11 \mathrm{C}(12)^{\mathrm{b}}$ & $+15 \mathrm{C}(12)^{\mathrm{b}}$ \\
\hline DCN superficial & Level & $\begin{array}{l}-15 C^{*}, \neq, b, c \\
-5 R(17)^{b}\end{array}$ & $\begin{array}{l}0 C^{\prime \neq, b, c} \\
+5 R(17)^{b}\end{array}$ & $\begin{array}{l}-7 R(17)^{b} \\
-6 C^{\ddagger, b, c}\end{array}$ & $+6 \mathrm{H}(8)^{\mathrm{b}}$ & $-14 \mathrm{H}(8)^{\mathrm{b}}$ & $-2 \mathrm{H}(9)^{\mathrm{b}}$ & $+12 \mathrm{C}(12)^{b}$ & $+1 \mathrm{C}(12)^{\mathrm{b}}$ & $+5 \mathrm{C}(12)^{\mathrm{b}}$ \\
\hline $\mathrm{CN}$ granular & Level & $\begin{array}{l}-18 C^{* \neq, b, c} \\
-3 R(17)^{b}\end{array}$ & $\begin{array}{l}+7 R(17)^{b} \\
+10 C^{\ddagger, b, c}\end{array}$ & $\begin{array}{l}O C^{\ddagger, b, c}, O R \\
(17)^{b}\end{array}$ & & & $-3 H(9)^{b}$ & & $-18 C^{*}(11)^{b}$ & \\
\hline LSO & Level & $+17 \mathrm{R}(17)^{\mathrm{b}}$ & $+4 \mathrm{R}(17)^{\mathrm{b}}$ & $-12 R(17)^{b}$ & & & & & & \\
\hline IC dorsal & Level & & & & & & $+2 \mathrm{H}(9)^{b}$ & & $-4 C^{\dagger, b}$ & \\
\hline IC ventral & Level & & & & & & $+3 \mathrm{H}(9)^{b}$ & & $+16 C^{\dagger, b}$ & \\
\hline IC lateral & Level & & & & & & $+3 \mathrm{H}(9)^{b}$ & & $-5 C^{\dagger, b}$ & \\
\hline MG total & Level & & & & & & & $-5 C^{\dagger, b}$ & $-12 C^{\dagger, b}$ & $-8 C^{\dagger, b}$ \\
\hline MG dorsal & Level & & & & & & $\mathrm{OH}(9)^{\mathrm{b}}$ & & & \\
\hline MG ventral & Level & & & & & & $-4 \mathrm{H}(9)^{\mathrm{b}}$ & & & \\
\hline MG medial & Level & & & & & & $-3 \mathrm{H}(9)^{b}$ & & & \\
\hline Aud Ctx total & Level & & & & & & & $-15 C^{*, \dagger, b}$ & $-6 C^{\dagger, b}$ & $-10 C^{\dagger, b}$ \\
\hline Aud Ctx layer I & Level & & & & & & $+5 \mathrm{H}(9)^{\mathrm{b}}$ & & & \\
\hline Aud Ctx layer II & Level & & & & & & $+5 \mathrm{H}(9)^{b}$ & & & \\
\hline Aud Ctx layer III & Level & & & & & & $+1 \mathrm{H}(9)^{\mathrm{b}}$ & & & \\
\hline Aud Ctx layer IV & Level & & & & & & $+4 \mathrm{H}(9)^{\mathrm{b}}$ & & & \\
\hline Aud Ctx layer V & Level & & & & & & $+5 \mathrm{H}(9)^{\mathrm{b}}$ & & & \\
\hline Aud Ctx layer VI & Level & & & & & & $+1 \mathrm{H}(9)^{\mathrm{b}}$ & & & \\
\hline
\end{tabular}

See Box 1 for table notes.

decreased in regions receiving sizable innervation from auditory nerve fibers. Large decreases in glutamate transporter (synthesis) in VCN (AVCN + PVCN) following kanamycin damage in guinea pigs correlated with decreased glutamate levels after cochlear ablation. Glutamate level and synthesis data in VCN after carboplatin resembled those for cochlear ablation except for a slower progression. To some extent, this slower progression paralleled a slower progression of the cochlear damage following carboplatin administration (12). At the mid time, inner hair cell loss was partial. In one chinchilla, where the loss of inner hair cells was largest in an intermediate portion of the cochlear spiral, the decrease in glutamate level was larger in an intermediate portion of the PVCN than in more dorsal or ventral locations (Figure 2). The effects of intense sound were more complex. In contrast to the data for other types of cochlear damage, glutamate levels increased in all $\mathrm{CN}$ regions at long times after intense sound. Although glutamate uptake decreased, as with cochlear ablation, release increased at all measured survival times. Receptors in the chinchilla VCN increased greatly at longer times after intense sound. A non-quantitative immunohistochemical study (not included in Table 1) reported a redistribution of $N$-methyl-D-aspartate
(NMDA) type glutamate receptors, from mostly axo-somatic to mostly axo-dendritic locations, in the $\mathrm{VCN}$ at various times up to more than a year after cochlear ablation (91). Another immunohistochemical study (not included in Table 1 because non-quantitative) found a loss of vesicular glutamate transporter (vGLUT1) from auditory nerve terminals and its appearance in VCN neuron somata 3 days after mechanical ablation of cochlear hair cells (92).

For more central auditory regions after cochlear ablation or carboplatin, many changes for glutamate were increases, unlike corresponding effects in the $\mathrm{CN}$. As in the $\mathrm{CN}$, glutamate levels increased in almost all central auditory regions at long times after intense sound.

Decreases in glutamate chemistry in the $\mathrm{CN}$ coupled with degeneration of auditory nerve fibers are consistent with evidence that glutamate serves as transmitter of auditory nerve fibers (3035). The mixed neurochemical changes of glutamate in the more central auditory regions after cochlear ablation or ototoxic damage may correlate with the evidence that glutamate serves as a neurotransmitter of various ascending (35), interneuronal (36), and descending $(37,38)$ pathways. These pathways would undergo 
Table 4 | GABA.

\begin{tabular}{|c|c|c|c|c|c|c|c|c|c|c|c|c|}
\hline \multirow[t]{2}{*}{ Region } & \multirow[t]{2}{*}{ Measurement } & \multicolumn{3}{|c|}{ Cochlear ablation } & \multicolumn{3}{|c|}{ Intense sound } & \multicolumn{3}{|c|}{ Carboplatin } & \multirow{2}{*}{$\frac{\text { Neomycin }}{\text { Mid }}$} & \multirow{2}{*}{$\begin{array}{c}\text { Salicylate } \\
\text { Long }\end{array}$} \\
\hline & & Short & Mid & Long & Short & Mid & Long & Short & Mid & Long & & \\
\hline $\mathrm{CN}$ total & Synthesis & $-18 G *(44)^{h}$ & & & & & & & & & & \\
\hline \multirow[t]{3}{*}{ AVCN } & Level & $\begin{array}{l}-81 C^{* *, b, c} \\
-14 R(17)^{b}\end{array}$ & $\begin{array}{l}-17 R \\
(17)^{b} \\
-8 C^{\ddagger, b, c}\end{array}$ & $\begin{array}{l}-15 R \\
(17)^{b} \\
-14 C^{\ddagger, b, c}\end{array}$ & & & $+2 \mathrm{H}(9)^{\mathrm{b}}$ & $-5 C(12)^{b}$ & $-13 \mathrm{C}(12)^{\mathrm{b}}$ & $-18 \mathrm{C}(12)^{\mathrm{b}}$ & & \\
\hline & Release & $+8 G(51)$ & & & & & & & & & & \\
\hline & Uptake & $+20 \mathrm{G}(51)$ & & & & & & & & & & \\
\hline \multirow[t]{3}{*}{ PVCN } & Level & $\begin{array}{l}-41 C^{*} \neq, b, c \\
+5 R(17)^{b}\end{array}$ & $\begin{array}{l}-42 C^{* \neq, b, c} \\
-7 R(17)^{b}\end{array}$ & $\begin{array}{l}-26 C^{\ddagger, b, c} \\
+19 R^{*} \\
(17)^{b}\end{array}$ & $-7 \mathrm{H}(8)^{b}$ & & $+3 \mathrm{H}(9)^{\mathrm{b}}$ & $-18 C *(12)^{b}$ & $-22 C^{*}(12)^{b}$ & $-18 C^{*}(12)^{b}$ & & \\
\hline & Release & $+5 \mathrm{G}(51)$ & & & & & & & & & & \\
\hline & Uptake & $+9 G(51)$ & & & & & & & & & & \\
\hline \multirow[t]{2}{*}{ DCN total } & Release & $-2 \mathrm{G}(51)$ & & & & & & & & & & \\
\hline & Uptake & OG (51) & & & & & & & & & & \\
\hline DCN deep & Level & $\begin{array}{l}-15 C^{\ddagger, b, c} \\
O R(17)^{b}\end{array}$ & $\begin{array}{l}-6 C^{\ddagger, b, c}, \\
\text { OR }(17)^{b}\end{array}$ & $\begin{array}{l}-15 C^{\ddagger}, b, c \\
+11 R(17)^{b}\end{array}$ & $+3 \mathrm{H}(8)^{\mathrm{b}}$ & $+14 \mathrm{H}(8)^{b}$ & $\mathrm{OH}(9)^{\mathrm{b}}$ & $+22 \mathrm{C}(12)^{\mathrm{b}}$ & $-1 \mathrm{C}(12)^{\mathrm{b}}$ & $+11 \mathrm{C}(12)^{\mathrm{b}}$ & & \\
\hline $\begin{array}{l}\text { DCN } \\
\text { superficial }\end{array}$ & Level & $\begin{array}{l}-17 \mathrm{R}(17)^{\mathrm{b}} \\
+2 \mathrm{C}^{\ddagger, b, c}\end{array}$ & $\begin{array}{l}-20 R^{*} \\
(17)^{b} \\
-4 C^{\ddagger, b, c}\end{array}$ & $\begin{array}{l}-10 R \\
(17)^{b} \\
-8 C^{\ddagger}, b, c\end{array}$ & $\mathrm{OH}(8)^{\mathrm{b}}$ & $-3 \mathrm{H}(8)^{\mathrm{b}}$ & $+1 \mathrm{H}(9)^{\mathrm{b}}$ & $-1 \mathrm{C}(12)^{\mathrm{b}}$ & $-14 \mathrm{C}(12)^{\mathrm{b}}$ & $-20 C^{*}(12)^{b}$ & & \\
\hline $\begin{array}{l}\mathrm{CN} \\
\text { granular }\end{array}$ & Level & $\begin{array}{l}-6 C^{\ddagger, b, c} \\
-4 R(17)^{b}\end{array}$ & $\begin{array}{l}-13 R \\
(17)^{b} \\
+26 C^{\ddagger, b, c}\end{array}$ & $\begin{array}{l}-5 R(17)^{b} \\
+2 C^{\ddagger}, b, c\end{array}$ & & & $-2 \mathrm{H}(9)^{\mathrm{b}}$ & & $-24 C^{*}(11)^{b}$ & & & \\
\hline LSO & Level & $-42 R^{*}(17)^{b}$ & $\begin{array}{l}-48 R^{*} \\
\left(17^{b}\right)\end{array}$ & $\begin{array}{l}-47 R^{*} \\
(17)^{b}\end{array}$ & & & & & & & & \\
\hline \multirow[t]{2}{*}{ IC total } & Synthesis & $\begin{array}{l}-34 R^{*}(82)^{i} \\
-7 G^{*}(44)^{h}\end{array}$ & & $\begin{array}{l}-30 R^{*} \\
(82)^{i}\end{array}$ & $+9 \mathrm{R}(83)^{\mathrm{j}}$ & $-21 R(83)^{j}$ & & & & & & $+57 R^{*}(84)^{n}$ \\
\hline & Receptors & & & & $-1 \mathrm{R}(85)^{\mathrm{k}}$ & $+19 R^{*}(85)^{k}$ & & & & & & $-17 R^{*}(84)^{n}$ \\
\hline \multirow[t]{3}{*}{ IC dorsal } & Level & & & & & & $+9 \mathrm{H}(9)^{\mathrm{b}}$ & & $-20 C^{\dagger, b}$ & & & \\
\hline & Synthesis & & & & $\begin{array}{l}-13 R \\
(83)^{j}\end{array}$ & $-4 \mathrm{R}(83)^{\mathrm{j}}$ & & & & & & \\
\hline & Receptors & & & & $\begin{array}{l}-15 G \\
(55)^{l}, \text { OR } \\
(85)^{\mathrm{k}}\end{array}$ & $+24 R^{*}(85)^{k}$ & & & & & & $-15 R(84)^{n}$ \\
\hline \multirow[t]{5}{*}{ IC ventral } & Level & & & & & & $+11 \mathrm{H}^{*}(9)^{b}$ & & $+15 C^{\dagger, b}$ & & & \\
\hline & Synthesis & & & & $-11 \mathrm{R}(83)^{\mathrm{j}}$ & $-2 \mathrm{R}(83)^{\mathrm{j}}$ & & & & & & \\
\hline & Release & $+26 \mathrm{G} *(51)$ & +11G (51) & $\begin{array}{l}+39 G^{*} \\
(51)\end{array}$ & & & & & & & $-74 G(86)$ & \\
\hline & Uptake & $+6 \mathrm{G}(51)$ & $-5 G(51)$ & $\begin{array}{l}-29 G^{*} \\
(51)\end{array}$ & & & & & & & & \\
\hline & Receptors & & & & $\begin{array}{l}-36 G^{*} \\
(55)^{l},-2 R \\
(85)^{k}\end{array}$ & $+22 \mathrm{R}(85)^{\mathrm{k}}$ & & & & & & $-22 R^{*}(84)^{n}$ \\
\hline \multirow[t]{3}{*}{ IC lateral } & Level & & & & & & $+11 \mathrm{H}^{*}(9)^{\mathrm{b}}$ & & $-15 C^{\dagger, b}$ & & & \\
\hline & Synthesis & & & & $\begin{array}{l}-12 R \\
(83)^{\mathrm{i}}\end{array}$ & $+3 R(83)^{j}$ & & & & & & \\
\hline & Receptors & & & & $\begin{array}{l}-13 G \\
(55)^{\prime},+9 R \\
(85)^{k}\end{array}$ & $+20 \mathrm{R}(85)^{\mathrm{k}}$ & & & & & & \\
\hline MG total & level & & & & & & & $-13 C^{\dagger, b}$ & $-22 C^{*}, \dagger, b$ & $-15 C^{\dagger, b}$ & & \\
\hline MG dorsal & Level & & & & & & $-6 \mathrm{H}(9)^{\mathrm{b}}$ & & & & & \\
\hline MG ventral & Level & & & & & & $+2 \mathrm{H}(9)^{\mathrm{b}}$ & & & & & \\
\hline MG medial & Level & & & & & & $+12 \mathrm{H}(9)^{\mathrm{b}}$ & & & & & \\
\hline
\end{tabular}


Table 4 | Continued

\begin{tabular}{|c|c|c|c|c|c|c|c|c|c|c|c|c|}
\hline \multirow[t]{2}{*}{ Region } & \multirow[t]{2}{*}{ Measurement } & \multicolumn{3}{|c|}{ Cochlear ablation } & \multicolumn{3}{|c|}{ Intense sound } & \multicolumn{3}{|c|}{ Carboplatin } & \multirow{2}{*}{$\frac{\text { Neomycin }}{\text { Mid }}$} & \multirow{2}{*}{$\begin{array}{c}\text { Salicylate } \\
\text { Long }\end{array}$} \\
\hline & & Short & Mid & Long & Short & Mid & Long & Short & Mid & Long & & \\
\hline Aud Ctx total & $\begin{array}{l}\text { Level } \\
\text { Synthesis }\end{array}$ & & & & $\begin{array}{l}-57 R^{*}(87)^{m} \\
-32 R^{*}(87)\end{array}$ & & & $-22 C^{\dagger, b}$ & $-14 C^{\dagger, b}$ & $-20 C^{\dagger, b}$ & & \\
\hline Aud Ctx layer I & Level & & & & & & $+1 \mathrm{H}(9)^{\mathrm{b}}$ & & & & & \\
\hline Aud Ctx layer II & Level & & & & & & $+1 \mathrm{H}(9)^{\mathrm{b}}$ & & & & & \\
\hline Aud Ctx layer III & Level & & & & & & $+6 \mathrm{H}(9)^{\mathrm{b}}$ & & & & & \\
\hline Aud Ctx layer IV & Level & & & & & & $+2 \mathrm{H}(9)^{\mathrm{b}}$ & & & & & \\
\hline Aud Ctx layer V & Level & & & & & & $+7 \mathrm{H}(9)^{\mathrm{b}}$ & & & & & \\
\hline Aud Ctx layer VI & Level & & & & & & $+2 \mathrm{H}(9)^{\mathrm{b}}$ & & & & & \\
\hline
\end{tabular}

See Box 1 for table notes.

relatively little physical degeneration after cochlear damage but may undergo complex compensatory changes. On the other hand, the effects on glutamate neurotransmission in the central auditory system at long times after intense sound differ from those of cochlear ablation and carboplatin administration. Under these conditions, glutamate is present at higher levels and is released more efficiently to receptors that are more sensitive; whereas glutamate is removed (uptake) from receptors less efficiently. All these changes together would make the central auditory system more excitable, which could result in or contribute to tinnitus.

\section{ASPARTATE}

Results for aspartate levels after cochlear ablation resemble those for glutamate levels with one notable, unexpected exception: in chinchillas, aspartate levels increased at short survival times (Table 2). Decreases in aspartate level in the $\mathrm{CN}$ after carboplatin treatment were similar to those for glutamate except that they developed more rapidly. At long survival times after intense sound, aspartate levels were consistently and often significantly increased, more so than glutamate levels, in all central auditory regions. Thus, for aspartate, even more so than for glutamate, the changes at long times after intense sound were opposite to those after cochlear ablation or carboplatin.

Parallel changes of aspartate neurochemistry are consistent with its close metabolic relationship with glutamate $(39,40)$. Increases in levels of aspartate at long times after intense sound exposure, combined with its ease of conversion to glutamate through the activity of aspartate aminotransferase (40), would contribute to a greater excitability of the central auditory system.

\section{GLUTAMINE}

Although glutamine is a major precursor for synthesis of glutamate, its changes after cochlear damage, except in the lateral superior olivary nucleus (LSO), were usually opposite to those of glutamate (Table 3). Intense sound damage resulted in no clear effect on glutamine levels. The most consistent effects were increased glutamine levels in VCN and deep DCN of rat after cochlear ablation and of chinchilla after carboplatin administration. Since glutamine levels are relatively high in glial cells $(42,56)$, these increases could reflect glial hypertrophy in the regions where auditory nerve fibers are degenerating.

\section{$\boldsymbol{\gamma}$-AMINOBUTYRIC ACID}

After cochlear ablation, there were striking decreases of GABA levels in chinchilla VCN [which were bilateral (16)], especially at shorter times (Table 4). In rat, there were [bilateral (17)] decreases in AVCN but not in PVCN. There were similar but less striking decreases in chinchilla VCN GABA levels after carboplatin (12). In superficial DCN, which receives little innervation from auditory nerve fibers $(93,94)$, GABA levels surprisingly decreased in rat after cochlear ablation and in chinchilla after carboplatin administration. One possible explanation for these decreases in GABA levels could be non-specific effects of trauma (95), which would be produced by cochlear damage, since GABA is not substantially related to auditory nerve fibers (17). A transneuronal effect of cochlear ablation on GABA levels in ipsilateral $\mathrm{CN}$ neurons is supported by an immunohistochemical study in rats (96). Counts of GABAimmunoreactive neurons decreased by $56 \%$ at 1 week and by $63 \%$ at 2 weeks after cochlear ablation, compared to contralateral, but there was no significant difference between the sides at 1 month (these data were not included in Table 4 due to uncertainty of sampling location within the $\mathrm{CN}$ ).

There were also consistent decreases of GABA level in the rat LSO ipsilateral to cochlear ablation, which might result from a retrograde effect on olivocochlear neurons after destruction of their terminals (17). Measurements for GABA, especially GABA receptors, usually showed decreases after ototoxic drug administration. The striking increase in GABA synthetic capacity in the IC after continuous, long-term salicylate administration in rats (84) might represent a compensatory response to the decrease in GABA receptors. After cochlear ablation, GABA synthesis and uptake decreased in the IC, whereas its release increased. These directions of change for release and uptake were similar to those for glutamate. In another study (not included in Table 4 due to uncertainty of sampling location within the IC), counts of GABAimmunoreactive neurons decreased by $33 \%$ in the contralateral IC at 1 week after unilateral cochlear ablation, but the decrease was not statistically significant at 1 month (97). Although GABA receptors and synthesis usually decreased in the IC at short times after intense sound, GABA receptors and levels increased at mid and long times, respectively. Similarly, GABA levels showed some tendency to increase in the medial geniculate (MG) and auditory cortex (Aud Ctx) at long times after intense sound. 
Lee and Godfrey

Central auditory neurotransmitter chemistry

Table 5 | Glycine.

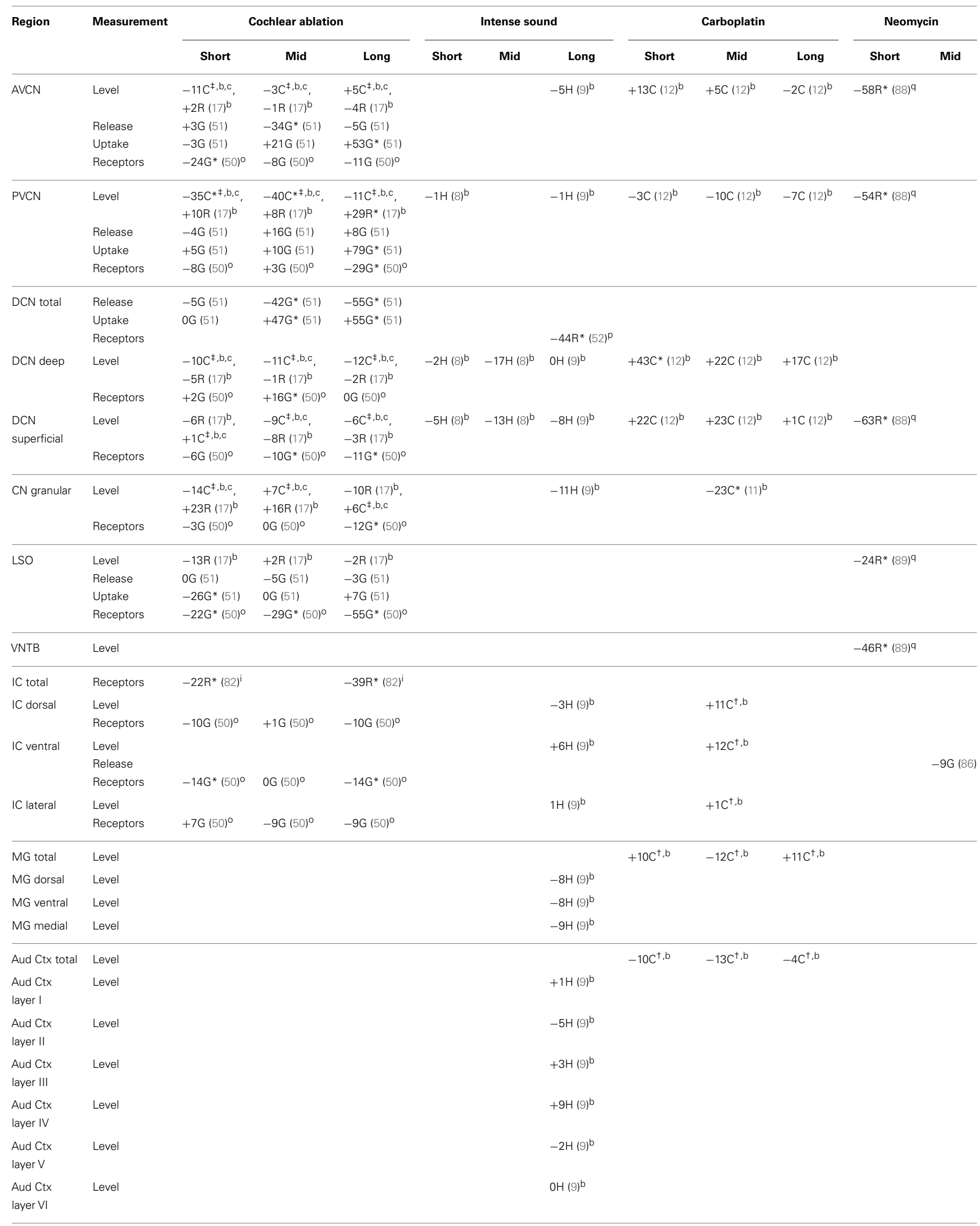

See Box 1 for table notes.

www.frontiersin.org

November 2014 | Volume 5 | Article 227 | 9 
Table 6 | Taurine.

\begin{tabular}{|c|c|c|c|c|c|c|c|c|c|c|}
\hline \multirow[t]{2}{*}{ Region } & \multirow[t]{2}{*}{ Measurement } & \multicolumn{3}{|c|}{ Cochlear ablation } & \multicolumn{3}{|c|}{ Intense sound } & \multicolumn{3}{|c|}{ Carboplatin } \\
\hline & & Short & Mid & Long & Short & Mid & Long & Short & Mid & Long \\
\hline AVCN & Level & $\begin{array}{l}-24 C^{* \neq, b, c}, \\
+14 R(17)^{b}\end{array}$ & $\begin{array}{l}+12 C^{\ddagger}, b, c \\
+14 R^{*}(17)^{b}\end{array}$ & $\begin{array}{l}+14 C^{\ddagger, b, c} \\
+25 R^{*}(17)^{b}\end{array}$ & & & $-9 \mathrm{H}(9)^{\mathrm{b}}$ & $+17 \mathrm{C}(12)^{\mathrm{b}}$ & $+19 \mathrm{C}(12)^{\mathrm{b}}$ & $+34 \mathrm{C}(12)^{\mathrm{b}}$ \\
\hline PVCN & Level & $\begin{array}{l}-53 C^{* \neq, b, c}, \\
+5 R(17)^{b}\end{array}$ & $\begin{array}{l}-53 C^{* \neq, b, c}, \\
+23 R(17)^{b}\end{array}$ & $\begin{array}{l}-10 C^{\ddagger, b, c} \\
+61 R^{*}(17)^{b}\end{array}$ & $-7 \mathrm{H}(8)^{b}$ & & $-13 H^{*}(9)^{b}$ & $-10 C(12)^{b}$ & $+14 \mathrm{C}(12)^{\mathrm{b}}$ & $+25 \mathrm{C}(12)^{b}$ \\
\hline DCN deep & Level & $\begin{array}{l}-12 C^{\ddagger, b, c}, \\
+1 R(17)^{b}\end{array}$ & $\begin{array}{l}-4 C^{\ddagger, b, c} \\
+9 R(17)^{b}\end{array}$ & $\begin{array}{l}-10 C^{\ddagger, b, c} \\
+24 R(17)^{b}\end{array}$ & $-11 \mathrm{H}(8)^{\mathrm{b}}$ & $-21 H^{*}(8)^{b}$ & $-10 \mathrm{H}(9)^{b}$ & $+3 \mathrm{C}(12)^{\mathrm{b}}$ & $+13 C(12)^{b}$ & $+25 C^{*}(12)^{b}$ \\
\hline DCN superficial & Level & $\begin{array}{l}-12 R(17)^{b}, \\
O C^{\ddagger, b, c}\end{array}$ & $\begin{array}{l}-6 R(17)^{b} \\
+3 C^{\ddagger}, b, c\end{array}$ & $\begin{array}{l}+3 C^{\ddagger, b, c} \\
+8 R(17)^{b}\end{array}$ & $-12 \mathrm{H}^{*}(8)^{b}$ & $-21 \mathrm{H}(8)^{\mathrm{b}}$ & $-7 \mathrm{H}(9)^{b}$ & $+2 \mathrm{C}(12)^{\mathrm{b}}$ & $+9 \mathrm{C}(12)^{\mathrm{b}}$ & $+7 \mathrm{C}(12)^{\mathrm{b}}$ \\
\hline $\mathrm{CN}$ granular & Level & $\begin{array}{l}-21 C^{\ddagger, b, c}, \\
+4 R(17)^{b}\end{array}$ & $\begin{array}{l}-1 R(17)^{b} \\
+13 C^{\ddagger, b, c}\end{array}$ & $\begin{array}{l}-12 R(17)^{b} \\
+1 C^{\ddagger, b, c}\end{array}$ & & & $-10 \mathrm{H}(9)^{\mathrm{b}}$ & & $-7 C(11)^{b}$ & \\
\hline LSO & Level & $-6 \mathrm{R}(17)^{\mathrm{b}}$ & OR $(17)^{b}$ & $-2 \mathrm{R}(17)^{\mathrm{b}}$ & & & & & & \\
\hline IC dorsal & Level & & & & & & $-12 H^{*}(9)^{b}$ & & $+17 C^{\dagger, b}$ & \\
\hline IC ventral & Level & & & & & & $-6 H^{*}(9)^{b}$ & & $+34 C^{\dagger, b}$ & \\
\hline IC lateral & Level & & & & & & $-6 \mathrm{H}(9)^{b}$ & & $+31 C^{\dagger, b}$ & \\
\hline MG total & Level & & & & & & & $+26 C^{*, \dagger, b}$ & $+21 C^{\dagger, b}$ & $+41 C * \dagger, b$ \\
\hline MG dorsal & Level & & & & & & $-11 \mathrm{H}(9)^{b}$ & & & \\
\hline MG ventral & Level & & & & & & $-13 H^{*}(9)^{b}$ & & & \\
\hline MG medial & Level & & & & & & $-4 \mathrm{H}(9)^{\mathrm{b}}$ & & & \\
\hline Aud Ctx total & Level & & & & & & & $+44 C^{*, t, b}$ & $+46 C^{*}, t, b$ & $+59 C^{*}, t, b$ \\
\hline Aud Ctx layer I & Level & & & & & & $-3 \mathrm{H}(9)^{\mathrm{b}}$ & & & \\
\hline Aud Ctx layer II & Level & & & & & & $-4 \mathrm{H}(9)^{b}$ & & & \\
\hline Aud Ctx layer III & Level & & & & & & $-4 \mathrm{H}(9)^{\mathrm{b}}$ & & & \\
\hline Aud Ctx layer IV & Level & & & & & & $-5 H(9)^{b}$ & & & \\
\hline Aud Ctx layer V & Level & & & & & & $-6 \mathrm{H}(9)^{\mathrm{b}}$ & & & \\
\hline Aud Ctx layer VI & Level & & & & & & $-9 H(9)^{b}$ & & & \\
\hline
\end{tabular}

See Box 1 for table notes.

Overall, the changes in the neurochemistry of GABA after cochlear damage do not consistently support a simultaneous correlation between loss of GABA inhibition and tinnitus.

\section{GLYCINE}

In the central auditory system, up through the IC, glycine receptors were almost always decreased at all times after cochlear ablation (Table 5). There are some striking contrasts, such as that between increased uptake and decreased release in AVCN and DCN total, and that between decreased levels in chinchilla PVCN and increased levels in rat PVCN. In both deep and superficial portions of the DCN, glycine levels decreased slightly after cochlear ablation but increased after carboplatin administration. These directions of change resembled those for glutamine in chinchillas but were opposite to those for glutamate and aspartate. At short times after neomycin administration, large decreases in density of glycine-immunoreactive puncta in the $\mathrm{CN}$ were localized on specific neuron types including spherical bushy cells, globular bushy cells, and radiate cells in the VCN and fusiform cells in the DCN (88). These decreases in numbers of glycineimmunoreactive puncta in rat $\mathrm{CN}$ (88), and also superior olive
(89), were much more striking than decreases in measured glycine levels in the same regions after cochlear ablation. This suggests compensating increases of glycine levels in some structures besides puncta, such as in reacting glial cells (67-69). In cerebellar cultures, glial cells have been reported to contain higher glycine levels than neurons (98). The only major change in glycine chemistry after intense sound was a prominent decrease of receptors in DCN total (52). In this same study, immunohistochemistry for glycine receptor subunits suggested that these decreases were most prominent at fusiform cells. Thus, the increased spontaneous activity of fusiform cells after intense sound exposure, which has been associated with tinnitus $(99,100)$, may at least partially result from decreased inhibitory input because of less glycine receptors (52) and maybe also less glycine neurotransmitter levels (9).

\section{TAURINE}

In regions of the $\mathrm{CN}$ that are well innervated by auditory nerve fibers, taurine levels were consistently increased in rat after cochlear ablation, but effects in chinchilla were mixed (Table 6). After carboplatin administration, there were increased taurine levels in all regions of the chinchilla $\mathrm{CN}$ except the granular region 
Table 7 | Acetylcholine.

\begin{tabular}{|c|c|c|c|c|c|c|c|c|c|c|}
\hline \multirow[t]{2}{*}{ Region } & \multirow[t]{2}{*}{ Measurement } & \multicolumn{3}{|c|}{ Cochlear ablation } & \multicolumn{3}{|c|}{ Intense sound } & \multicolumn{3}{|c|}{ Carboplatin } \\
\hline & & Short & Mid & Long & Short & Mid & Long & Short & Mid & Long \\
\hline \multirow[t]{2}{*}{ AVCN } & Synthesis & $\begin{array}{l}+19 R(14)^{b} \text { and } \\
+91 R^{*}(60)^{r}\end{array}$ & $+54 R^{*}(14)^{b}$ & $-4 \mathrm{R}(14)^{\mathrm{b}}$ & $+252 \mathrm{H}^{*}(7)^{\mathrm{b}}$ & & $+28 \mathrm{H}\left(7^{b}\right)$ and $-23 \mathrm{H}(10)^{b}$ & & & \\
\hline & Receptors & $+6 \mathrm{R}(15)^{\mathrm{S}}$ & $+28 R^{*}(15)^{\mathrm{s}}$ & $+67 R^{*}(15)^{\mathrm{s}}$ & & & & & & \\
\hline \multirow[t]{2}{*}{ PVCN } & Synthesis & $+37 R^{*}(14)^{b}$ & $+54 R^{*}(14)^{b}$ & $+40 R^{*}(14)^{b}$ & $+27 \mathrm{H}(7)^{\mathrm{b}}$ & & $+27 \mathrm{H}(7)^{b}$ & & $-32 C^{\dagger, b}$ & \\
\hline & Receptors & $-15 R^{*}(15)^{S}$ & $+18 \mathrm{R}(15)^{\mathrm{s}}$ & $+70 R^{*}(15)^{\mathrm{s}}$ & & & & & & \\
\hline \multirow[t]{2}{*}{ DCN deep } & Synthesis & $+8 \mathrm{R}(14)^{\mathrm{b}}$ & $+25 \mathrm{R}(14)^{\mathrm{b}}$ & $+7 \mathrm{R}(14)^{\mathrm{b}}$ & $+37 \mathrm{H}(7)^{\mathrm{b}}$ & & $+36 \mathrm{H}(7)^{\mathrm{b}}$ and $-21 \mathrm{H}(10)^{\mathrm{b}}$ & & $+9 c^{\dagger, b}$ & \\
\hline & Receptors & $+7 \mathrm{R}(15)^{\mathrm{s}}$ & $+20 R^{*}(15)^{s}$ & $+22 R^{*}(15)^{\mathrm{s}}$ & & & & & & \\
\hline \multirow[t]{2}{*}{ DCN superficial } & Synthesis & $-3 \mathrm{R}(14)^{\mathrm{b}}$ & $+15 \mathrm{R}(14)^{\mathrm{b}}$ & $-9 \mathrm{R}(14)^{\mathrm{b}}$ & $+35 \mathrm{H}(7)^{\mathrm{b}}$ & & $+16 \mathrm{H}\left(7^{\mathrm{b}}\right)$ and $-11 \mathrm{H}(10)^{\mathrm{b}}$ & & $+62 C^{\dagger, b}$ & \\
\hline & Receptors & $+3 R(15)^{S}$ & $+8 \mathrm{R}(15)^{\mathrm{s}}$ & $+11 R^{*}(15)^{\mathrm{s}}$ & & & & & & \\
\hline \multirow[t]{2}{*}{$\mathrm{CN}$ granular } & Synthesis & $-24 R(14)^{b}$ & $+49 R^{*}(14)^{b}$ & $-5 R(14)^{b}$ & $+38 \mathrm{H}^{*}(7)^{\mathrm{b}}$ & & $+28 \mathrm{H}(7)^{\mathrm{b}}$ and $+38 \mathrm{H}^{*}(10)^{\mathrm{b}}$ & & $-46 \mathrm{C}^{\dagger, \mathrm{b}}$ & \\
\hline & Receptors & $+1 \mathrm{R}(15)^{\mathrm{s}}$ & $+11 R^{*}(15)^{s}$ & $+27 R^{*}(15)^{s}$ & & & & & & \\
\hline LSO & Synthesis & $-25 R^{*}(14)^{b}$ & $-5 R(14)^{b}$ & $-37 R^{*}(14)^{b}$ & & & $+40 \mathrm{H}^{*}(10)^{\mathrm{b}}$ & & & \\
\hline VNTB & Synthesis & $+19 \mathrm{R}(14)^{\mathrm{b}}$ & $-19 \mathrm{R}(14)^{\mathrm{b}}$ & $-22 R^{*}(14)^{b}$ & & & $+32 \mathrm{H}(10)^{\mathrm{b}}$ & & & \\
\hline IC dorsal & Synthesis & & & & & & $+10 \mathrm{H}(10)^{b}$ & & & \\
\hline IC ventral & Synthesis & & & & & & $-12 \mathrm{H}(10)^{\mathrm{b}}$ & & & \\
\hline IC lateral & Synthesis & & & & & & $\mathrm{OH}(10)^{b}$ & & & \\
\hline Aud Ctx layer I & Synthesis & & & & & & $+1 \mathrm{H}(10)^{b}$ & & & \\
\hline Aud Ctx layer II & Synthesis & & & & & & $-7 H(10)^{b}$ & & & \\
\hline Aud Ctx layer III & Synthesis & & & & & & $+2 \mathrm{H}(10)^{b}$ & & & \\
\hline Aud Ctx layer IV & Synthesis & & & & & & $-6 \mathrm{H}(10)^{\mathrm{b}}$ & & & \\
\hline Aud Ctx layer V & Synthesis & & & & & & $+1 \mathrm{H}(10)^{b}$ & & & \\
\hline Aud Ctx layer VI & Synthesis & & & & & & $+3 \mathrm{H}(10)^{\mathrm{b}}$ & & & \\
\hline
\end{tabular}

See Box 1 for table notes.

$(11,12)$ and consistent increases in more central regions. Increases of taurine after cochlear damage in regions densely innervated by the auditory nerve could, as with glutamine, be related to glial hypertrophy in regions where auditory nerve fibers are degenerating, since taurine concentrations are relatively high in glia (42, 56), but this would not account for increases in more central auditory regions. Intense sound led at long times to slight-tomoderate decreases of taurine levels in all regions of the hamster central auditory system $(8,9)$. These changes in taurine levels were almost always opposite to those for aspartate. Because of taurine's association with GABA and glycine neurotransmission (57-59), decreased taurine levels could be associated with decreased inhibitory activity in the central auditory system, which could result in tinnitus. Previous animal studies have found that a decreased blood taurine concentration is associated with hearing loss (101) and that taurine administration can decrease behavioral evidence of tinnitus (102).

\section{ACETYLCHOLINE}

In the $\mathrm{CN}$ after cochlear ablation, the synthetic capacity for acetylcholine (choline acetyltransferase activity) increased at mid times, but it returned toward control levels in most regions at long times (Table 7). Muscarinic acetylcholine receptors increased in CN regions at mid and long times after cochlear ablation. Synthetic capacity for acetylcholine increased in all $\mathrm{CN}$ regions through 2 months after intense sound, but the increase was not maintained through 5 months except in the granular region. Some of these changes may correlate with formation of new synapses after acoustic trauma (73). The sustained increase of choline acetyltransferase activity in the $\mathrm{CN}$ granular region $(7,10)$, as well as an increase in muscarinic acetylcholine receptor sensitivity in the superficial DCN (103), could be consistent with formation of new cholinergic synapses or upregulation of existing cholinergic synapses upon granule cells. Since many granule cells form excitatory glutamatergic synapses with fusiform and cartwheel cells in the superficial DCN $(36,104)$, increased cholinergic activity, leading to increased granule cell activity, could change the balance of excitatory and inhibitory input to DCN fusiform cells and thereby alter their activity $(10,105-107)$. Increased spontaneous activity of DCN fusiform cells after acoustic trauma has been associated with tinnitus $(99,100)$.

Acetylcholine synthetic capacity in the LSO and the ventral nucleus of the trapezoid body (VNTB) decreased after cochlear ablation, perhaps as a retrograde effect of olivocochlear terminal destruction (14), but it increased at 5 months after intense sound exposure. Since these two regions give rise to the cholinergic olivocochlear bundle (108) and olivo-CN connections that terminate in CN granular regions (109), the increased choline acetyltransferase activities in the LSO and VNTB at long times after intense sound exposure, together with the increased activities in the $\mathrm{CN}$ granular region, may suggest an upregulation of olivo-CN projections. 


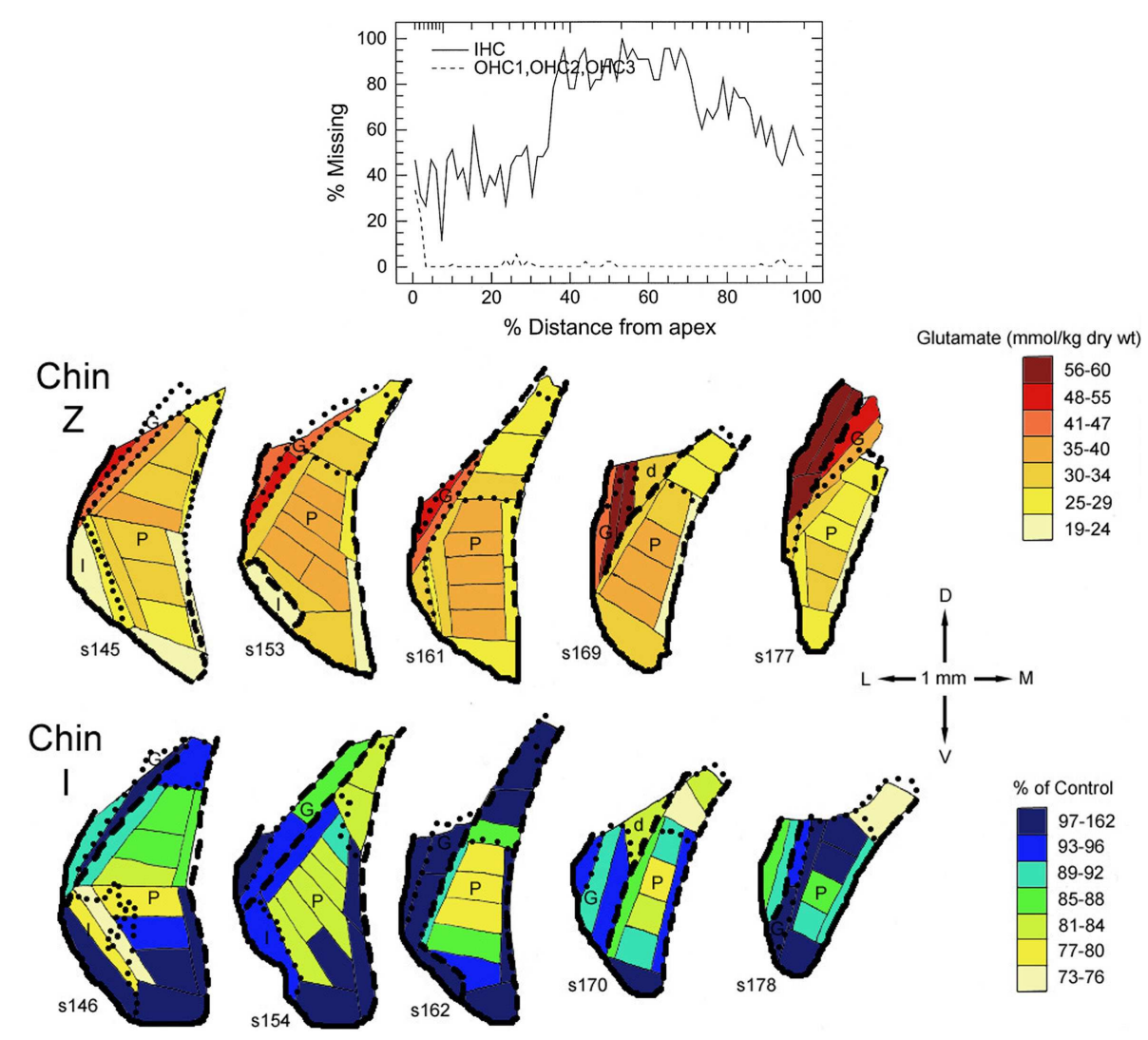

FIGURE 2 | Maps of glutamate levels in the posteroventral cochlear nucleus (PVCN) of a control chinchilla (Chin Z) and of a chinchilla treated 29 days earlier with $100 \mathrm{mg} / \mathbf{k g}$ carboplatin by intraperitoneal injection (Chin I). The loss of cochlear inner ( $\mathrm{IHC})$ and outer $(\mathrm{OHC})$ hair cells is plotted in the diagram at the top vs. distance from the cochlear apex. Glutamate levels are color coded for Chin Z. For Chin I, the glutamate levels are expressed as percentages of those for comparable locations in Chin Z. For detailed methods, see Godfrey et al. (12).

\section{SEROTONIN AND NOREPINEPHRINE}

Relatively, little work has been published concerning changes in other neurotransmitter-related chemistry in the central auditory system after cochlear damage. Increases in serotonin and norepinephrine metabolites have been reported within $45 \mathrm{~min}$ after white noise exposure in rat caudal $\mathrm{CN}(\mathrm{DCN}+\mathrm{PVCN})$ and Aud Ctx (110). For the serotonin metabolite, there was a $34 \%$ increase in the $\mathrm{CN}$ and $22 \%$ increase in the Aud Ctx. For the norepinephrine metabolite, there was a $121 \%$ increase in the $\mathrm{CN}$, but no measurable change in the Aud Ctx. Since these changes occurred after very short times at $70 \mathrm{~dB}$ sound pressure level but not at 90 or $110 \mathrm{~dB}$, they represent increased metabolism but not effects of cochlear damage from acoustic trauma.

The effects of salicylate on the serotonin levels in the central auditory system were measured by microdialysis (111). Within few hours after systemic administration of salicylate, serotonin levels increased by about $170 \%$ in both the IC and Aud Ctx of rats. Another study investigated the effects of acoustic trauma on the density of serotonergic fibers (estimated by measures of serotonergic fiber length density using immunohistochemistry for serotonin reuptake transporter) in the IC of mice after 3-16 weeks (112). Decreases of 17, 10, and 14\% were reported in dorsal, lateral, and central regions of the IC on the affected (contralateral) side as compared to ipsilateral. Although the measured changes in serotonin chemistry in these studies were in opposite directions at very different times and after different interventions, both would lead to an imbalance of neurotransmitter chemistry in the central auditory system, which might be associated with tinnitus. There is some evidence that changes in serotonin neurotransmission might have a role in tinnitus $(2,113,114)$.

\section{DISCUSSION \\ CLINICAL APPLICATIONS}

The compiled results from available studies suggest that different types of cochlear damage may lead to different neurotransmitterrelated chemical changes in the central auditory system, even though they all could result in tinnitus. The changes following ototoxic drug administration resemble those after cochlear ablation, although with a slower development and smaller magnitude, but the changes at long times after intense sound tend to be in the opposite direction. This implies that tinnitus may result from a variety of different, even contrasting, chemical changes. Further, the neurochemical changes reported for several different transmitter systems after cochlear damage suggest that imbalances involving any of the various transmitter systems could result in tinnitus. Perhaps, more detailed investigations would distinguish 
different types of tinnitus. It may also be useful to distinguish between tinnitus as a symptom, which commonly occurs acutely after peripheral insult or even spontaneously, and tinnitus as a chronic morbidity. Although it is reasonable to hypothesize that tinnitus may result from an increase in excitatory neurotransmitter chemistry and/or a decrease in inhibitory neurotransmitter chemistry, the available data suggest that the chemical basis for tinnitus may not depend on the direction of change in these chemistries so much as any imbalance between them. Another factor to consider is the magnitude of cochlear damage. Perhaps, the similarity of chemical effects after cochlear ablation and at longer times after carboplatin administration is related to the magnitude of inner hair cell loss, which has been proposed as the most important factor that leads to tinnitus (115). Cochlear ablation leads to total hair cell loss and carboplatin administration to almost total inner hair cell loss $(11,12)$, whereas intense tone exposure leads to more limited inner hair cell damage $(73,116)$. More detailed measurements of damage magnitudes may result in a clearer understanding of subsequent chemical changes.

A factor that confounds the application of results for animal experiments to human hearing loss and tinnitus is the variation in experimental results among animal species. The most consistent effect found was the decrease in glutamate and aspartate levels and glutamate release and uptake in the $\mathrm{CN}$ regions receiving major innervation from the auditory nerve following cochlear ablation. Even for this effect, however, if one only studied short post-damage survival times, the surprising increase of aspartate levels at these times in chinchillas would complicate interpretations of the data. Collection of data for a wide range of post-damage survival times showed that some apparent contradictions between the results for different species result from different time courses of the chemical changes after cochlear damage rather than major differences in the ultimate direction of the change.

\section{NEED FOR MORE DATA}

It is evident from the tables that most available neurotransmitterrelated chemical data for the central auditory system after cochlear damage have been obtained for the $\mathrm{CN}$ and IC, and there is a relative lack of data for the MG and Aud Ctx. Further study at different levels of the central auditory system is needed. There is also a relative lack of data for effects of ototoxic drugs. Besides carboplatin, there are over 200 medications of several types that can adversely affect hearing (3). This underscores the need for more research on the effects of various drugs on the neurochemistry of the central auditory system.

\section{FUTURE DIRECTIONS}

The chemical effects of cochlear damage following intense sound or ototoxic drugs generally appear to develop more slowly than those following the more severe damage produced by surgical ablation of the cochlea. It may therefore be useful to expand the time frame for measuring chemical changes after intense sound or ototoxic drugs in order to identify changes that underlie chronic tinnitus. In the case of intense sound, the changes in amino acid levels found 5 months after the exposure were more consistent for several amino acids than those at times $<2$ months after exposure.
The changes in choline acetyltransferase activity (for acetylcholine synthesis) in the $\mathrm{CN}$ remained significant only in the granular region at 5 months after exposure.

Some amino acids such as aspartate and taurine may be associated with tinnitus indirectly through metabolic or functional relationships to neurotransmitters. Additional studies on such amino acids may prove useful for understanding the neurochemical basis of tinnitus.

Perhaps because of various experimental limitations, such as insufficient sample size, small chemical changes in a given region may not reach statistical significance. However, patterns of change that are widespread over many regions might indicate functionally important neurochemical effects underlying tinnitus. Studies with larger sample sizes may improve the likelihood of showing statistically significant changes, but even data not reaching statistical significance may still contribute to the understanding of the chemical mechanisms associated with tinnitus.

The studies investigating the neurochemical changes underlying tinnitus comprise one component of a large body of tinnitusrelated research. Progressive scientific advances in this component may contribute toward greatly needed improvements in the prevention, diagnosis, and management of tinnitus.

\section{AUTHOR CONTRIBUTIONS}

Both authors contributed to all aspects of this work.

\section{ACKNOWLEDGMENTS}

Support for this work was received from the University of Toledo Foundation and the American Tinnitus Association. We are grateful to Dr. Abraham Lee for helpful comments on the manuscript and to Dr. Hongyan Li for assistance with translation of articles written in Chinese.

\section{REFERENCES}

1. Henry JA, Dennis KC, Schechter MA. General review of tinnitus: prevalence, mechanisms, effects, and management. J Speech Lang Hear Res (2005) 48:1205-35. doi:10.1044/1092-4388(2005/084)

2. Eggermont JJ. The Neuroscience of Tinnitus. Oxford: Oxford University Press (2012).

3. Fausti SA, Wilmington DJ, Helt PV, Helt WJ, Konrad-Martin D. Hearing health and care: the need for improved hearing loss prevention and hearing conservation practices. J Rehabil Res Dev (2005) 42:45-62. doi:10.1682/JRRD.2005. 02.0039

4. Veterans Benefits Administration. Annual Benefits Reports for FY2013 [Database on the Internet]. Washington, DC: U.S. Department of Veterans Affairs (2014). Available from: http://www.vba.va.gov/reports.htm

5. Gold JR, Bajo VM. Insult-induced adaptive plasticity of the auditory system. Front Neurosci (2014) 8:110. doi:10.3389/fnins.2014.00110

6. Bauer CA, Turner JG, Caspary DM, Myers KS, Brozoski TJ. Tinnitus and inferior colliculus activity in chinchillas related to three distinct patterns of cochlear trauma. J Neurosci Res (2008) 86:2564-78. doi:10.1002/jnr.21699

7. Jin Y-M, Godfrey DA, Wang J, Kaltenbach JA. Effects of intense tone exposure on choline acetyltransferase activity in the hamster cochlear nucleus. Hear Res (2006) 216-7:168-75. doi:10.1016/j.heares.2006.02.002

8. Godfrey DA, Mikesell NL, Godfrey TG, Fulcomer AB, Kong W, Godfrey MA, et al. Effects of high-intensity sound exposure on neurotransmitter chemistry in the central auditory system. Semin Hear (2008) 29:259-69. doi:10.1055/s-0028-1082032

9. Godfrey DA, Kaltenbach JA, Chen K, Ilyas O, Liu X, Licari F, et al. Amino acid concentrations in the hamster central auditory system and long-term effects of intense tone exposure. J Neurosci Res (2012) 90:2214-24. doi:10.1002/jnr.23095 
10. Godfrey DA, Kaltenbach JA, Chen K, Ilyas O. Choline acetyltransferase activity in the hamster central auditory system and long-term effects of intense tone exposure. J Neurosci Res (2013) 91:987-96. doi:10.1002/jnr.23227

11. Li Y, Godfrey DA, Godfrey MA, Ding D-L, Salvi R. Effects of carboplatin on amino acid chemistry in chinchilla cochlear nucleus. Hear Res (2002) 165:19-29. doi:10.1016/S0378-5955(01)00389-6

12. Godfrey DA, Godfrey MA, Ding D-L, Chen K, Salvi RJ. Amino acid concentrations in chinchilla cochlear nucleus at different times after carboplatin treatment. Hear Res (2005) 206:64-73. doi:10.1016/j.heares.2005.03.004

13. Dille MF, Konrad-Martin D, Gallun F, Helt WJ, Gordon JS, Reavis KM, et al. Tinnitus onset rates from chemotherapeutic agents and ototoxic antibiotics: results of a large prospective study. J Am Acad Audiol (2010) 21:409-17. doi:10.3766/jaaa.21.6.6

14. Jin Y-M, Godfrey DA, Sun Y. Effects of cochlear ablation on choline acetyltransferase activity in the rat cochlear nucleus and superior olive. J Neurosci Res (2005) 81:91-101. doi:10.1002/jnr.20536

15. Jin Y-M, Godfrey DA. Effects of cochlear ablation on muscarinic acetylcholine receptor binding in the rat cochlear nucleus. J Neurosci Res (2006) 83:157-66. doi:10.1002/jnr.20706

16. Godfrey DA, Chen K, Godfrey MA, Jin Y-M, Robinson KT, Hair C. Effects of cochlear ablation on amino acid concentrations in the chinchilla posteroventral cochlear nucleus, as compared to rat. Neuroscience (2008) 154:304-14. doi:10.1016/j.neuroscience.2007.12.031

17. Godfrey DA, Jin Y-M, Liu X, Godfrey MA. Effects of cochlear ablation on amino acid levels in the rat cochlear nucleus and superior olive. Hear Res (2014) 309:44-54. doi:10.1016/j.heares.2013.11.005

18. Berliner KI, Shelton C, Hitselberger WE, Luxford WM. Acoustic tumors: effect of surgical removal on tinnitus. Am J Otol (1992) 13:13-7.

19. Soussi T, Otto SR. Effects of electrical brainstem stimulation on tinnitus. Acta Otolaryngol (1994) 114:135-40. doi:10.3109/00016489409126031

20. Ryan AF, Axelsson GA, Woolf NK. Central auditory metabolic activity induced by intense noise exposure. Hear Res (1992) 61:24-30. doi:10.1016/03785955(92)90032-I

21. Muly SM, Gross JS, Morest DK, Potashner SJ. Synaptophysin in the cochlear nucleus following acoustic trauma. Exp Neurol (2002) 177:202-21. doi:10. 1006/exnr.2002.7963

22. Michler SA, Illing RB. Molecular plasticity in the rat auditory brainstem: modulation of expression and distribution of phosphoserine, phosphorCREB and TrkB after noise trauma. Audiol Neurootol (2003) 8:190-206. doi:10.1159/000071060

23. Zhang JS, Kaltenbach JA, Wang J, Bronchti G. Changes in $\left[{ }^{14} \mathrm{C}\right]$-2-deoxyglucose uptake in the auditory pathway of hamsters previously exposed to intense sound. Hear Res (2003) 185:13-21. doi:10.1016/S0378-5955(03)00276-4

24. Zhang JS, Kaltenbach JA, Wang J, Kim SA. Fos-like immunoreactivity in auditory and nonauditory brain structures of hamsters previously exposed to intense sound. Exp Brain Res (2003) 153:655-60. doi:10.1007/s00221-0031612-4

25. Rybak LP. Neurochemistry of the peripheral and central auditory system after ototoxic drug exposure: implications for tinnitus. Int Tinnitus J (2005) 11:23-30.

26. Tan J, Rüttiger L, Panford-Walsh R, Singer W, Schulze H, Kilian SB, et al. Tinnitus behavior and hearing function correlate with reciprocal expression patterns of BDNF and ARG3.1/arc in auditory neurons following acoustic trauma. Neuroscience (2007) 145:715-26. doi:10.1016/j.neuroscience.2006.11.067

27. Kraus KS, Ding D, Zhou Y, Salvi RJ. Central auditory plasticity after carboplatininduced unilateral inner ear damage in the chinchilla: up-regulation of GAP43 in the ventral cochlear nucleus. Hear Res (2009) 255:33-43. doi:10.1016/j. heares.2009.05.001

28. Kraus KS, Ding S, Jiang H, Lobarinas E, Sun W, Salvi RJ. Relationship between noise-induced hearing-loss, persistent tinnitus and growth-associated protein-43 expression in the rat cochlear nucleus: does synaptic plasticity in ventral cochlear nucleus suppress tinnitus? Neuroscience (2011) 194:309-25. doi:10.1016/j.neuroscience.2011.07.056

29. Hildebrandt H, Hoffmann NA, Illing RB. Synaptic reorganization in the adult rat's ventral cochlear nucleus following its total sensory deafferentation. PLoS One (2011) 6(8):e23686. doi:10.1371/journal.pone.0023686

30. Wenthold RJ, Gulley RL. Aspartic acid and glutamic acid levels in the cochlear nucleus after auditory nerve lesion. Brain Res (1977) 138:279-84.
31. Wenthold RJ. Glutamic acid and aspartic acid in subdivisions of the cochlear nucleus after auditory nerve lesion. Brain Res (1978) 143:544-8. doi:10.1016/ 0006-8993(78)90365-7

32. Wenthold RJ. Release of endogenous glutamic acid, aspartic acid and GABA from cochlear nucleus slices. Brain Res (1979) 162:338-43. doi:10.1016/00068993(79)90294-4

33. Wenthold RJ. Glutaminase and aspartate aminotransferase decrease in the cochlear nucleus after auditory nerve lesion. Brain Res (1980) 190:293-7. doi:10.1016/0006-8993(80)91183-X

34. Wenthold RJ, Hunter C, Petralia RS. Excitatory amino acid receptors in the rat cochlear nucleus. In: Merchán MA, Juiz JM, Godfrey DA, Mugnaini E, editors. The Mammalian Cochlear Nuclei Organization and Function. New York, NY: Plenum Press (1993). p. 179-94.

35. Godfrey DA, Parli JA, Dunn JD, Ross CD. Neurotransmitter microchemistry of the cochlear nucleus and superior olivary complex. In: Syka J, Masterton RB, editors. Auditory Pathway. New York, NY: Plenum Publishing Corporation (1988). p. 107-21.

36. Godfrey DA, Godfrey TG, Mikesell NL, Waller HJ, Yao W, Chen K, et al. Chemistry of granular and closely related regions of the cochlear nucleus. In: Syka J, editor. Acoustical Signal Processing in the Central Auditory System. New York, NY: Plenum Publishing Corporation (1997). p. 139-53.

37. Wright DD, Ryugo DK. Mossy fiber projections from the cuneate nucleus to the cochlear nucleus in the rat. J Comp Neurol (1996) 365:159-72. doi:10.1002/ (SICI) 1096-9861(19960129)365:1<159::AID-CNE12>3.0.CO;2-L

38. Zeng C, Nannapaneni N, Jianxun Z, Hughes LF, Shore S. Cochlear damage changes the distribution of vesicular glutamate transporters associated with auditory and nonauditory inputs to the cochlear nucleus. J Neurosci (2009) 29:4210-7. doi:10.1523/JNEUROSCI.0208-09.2009

39. Dingledine R, McBain CJ. Excitatory amino acid transmitters. In: Siegel GJ, Agranoff BW, Albers RW, Molinoff PB, editors. Basic Neurochemistry. New York, NY: Raven Press (1994). p. 367-87.

40. Ross CD, Godfrey DA, Parli JA. Amino acid concentrations and selected enzyme activities in rat auditory, olfactory, and visual systems. Neurochem Res (1995) 20:1483-90. doi:10.1007/BF00970598

41. Shank RP, Aprison MH. Present status and significance of the glutamine cycle in neural tissues. Life Sci (1981) 28:837-42. doi:10.1016/0024-3205(81)90044-8

42. Ottersen OP, Zhang N, Walberg F. Metabolic compartmentation of glutamate and glutamine: morphological evidence obtained by quantitative immunocytochemistry in rat cerebellum. Neuroscience (1992) 46:519-34. doi:10.1016/ 0306-4522(92)90141-N

43. Tachibana M, Kuriyama K. Gamma-aminobutyric acid in the lower auditory pathway of the guinea pig. Brain Res (1974) 69:370-4. doi:10.1016/00068993(74)90017-1

44. Fisher SK, Davies WE. GABA and its related enzymes in the lower auditory system of the guinea pig. J Neurochem (1976) 27:1145-55. doi:10.1111/j.14714159.1976.tb00321.x

45. Moore MJ, Caspary DM. Strychnine blocks binaural inhibition in lateral superior olivary neurons. J Neurosci (1983) 3:237-42.

46. Potashner SJ, Lindberg N, Morest DK. Uptake and release of gammaaminobutyric acid in the guinea pig cochlear nucleus after axotomy of cochlear and centrifugal fibers. J Neurochem (1985) 45:1558-66. doi:10.1111/j.14714159.1985.tb07227.x

47. Potashner SJ, Benson CG, Ostapoff E-M, Lindberg N, Morest DK. Glycine and GABA: transmitter candidates of projections descending to the cochlear nucleus. In: Merchán MA, Juiz JM, Godfrey DA, Mugnaini E, editors. The Mammalian Cochlear Nuclei Organization and Function. New York, NY: Plenum Press (1993). p. 195-210.

48. Potashner SJ, Suneja SK, Benson CG. Altered glycinergic synaptic activities in guinea pig brain stem auditory nuclei after unilateral cochlear ablation. Hear Res (2000) 147:125-36. doi:10.1016/S0378-5955(00)00126-X

49. Altschuler RA, Juiz JM, Shore SE, Bledsoe SC, Helfert RH, Wenthold RJ. Inhibitory amino acid synapses and pathways in the ventral cochlear nucleus. In: Merchán MA, Juiz JM, Godfrey DA, Mugnaini E, editors. The Mammalian Cochlear Nuclei Organization and Function. New York, NY: Plenum Press (1993). p. 211-24.

50. Suneja SK, Benson CG, Potashner SJ. Glycine receptors in adult guinea pig brain stem auditory nuclei: regulation after unilateral cochlear ablation. Exp Neurol (1998) 154:473-88. doi:10.1006/exnr.1998.6946 
51. Suneja SK, Potashner SJ, Benson CG. Plastic changes in glycine and GABA release and uptake in adult brain stem auditory nuclei after unilateral middle ear ossicle removal and cochlear ablation. Exp Neurol (1998) 151:273-88. doi:10.1006/exnr.1998.6812

52. Wang H, Brozoski TJ, Turner JG, Ling L, Parrish JL, Hughes LF, et al. Plasticity at glycinergic synapses in dorsal cochlear nucleus of rats with behavioral evidence of tinnitus. Neuroscience (2009) 164:747-59. doi:10.1016/j.neuroscience.2009. 08.026

53. Wang H, Brozoski TJ, Caspary DM. Inhibitory neurotransmission in animal models of tinnitus: maladaptive plasticity. Hear Res (2011) 279:111-7. doi:10.1016/j.heares.2011.04.004

54. Dong S, Mulders WH, Rodger J, Woo S, Robertson D. Acoustic trauma evokes hyperactivity and changes in gene expression in guinea-pig auditory brainstem. Eur J Neurosci (2010) 31:1616-28. doi:10.1111/j.1460-9568.2010. 07183.x

55. Dong S, Rodger J, Mulders WH, Robertson D. Tonotopic changes in GABA receptor expression in guinea pig inferior colliculus after partial unilateral hearing loss. Brain Res (2010) 1342:24-32. doi:10.1016/j.brainres. 2010.04.067

56. Hassel B, Westergaard N, Schousboe A, Fonnum F. Metabolic differences between primary cultures of astrocytes and neurons from cerebellum and cerebral cortex. Effects of fluorocitrate. Neurochem Res (1995) 20:413-20. doi:10.1007/BF00973096

57. Ottersen OP, Madsen S, Storm-Mathisen J, Somogyi P, Scopsi L, Larson LI. Immunocytochemical evidence suggests that taurine is colocalized with GABA in the Purkinje cell terminals, but that the stellate cell terminals predominantly contain GABA: a light- and electron microscopic study of the rat cerebellum. Exp Brain Res (1988) 72:407-16. doi:10.1007/BF00250262

58. Walberg F, Ottersen OP, Rinvik E. GABA, glycine, aspartate, glutamate and taurine in the vestibular nuclei: an immunocytochemical investigation in the cat. Exp Brain Res (1990) 79:547-63. doi:10.1007/BF00229324

59. Albrecht J, Schousboe A. Taurine interaction with neurotransmitter receptors in the CNS: an update. Neurochem Res (2005) 30:1615-21. doi:10.1007/s11064005-8986-6

60. Meidinger M, Hildebrandt-Schoenfeld H, Illing RB. Cochlear damage induces GAP-43 expression in cholinergic synapses of the cochlear nucleus in the adult rat: a light and electron microscopic study. Eur J Neurosci (2006) 23:3187-99. doi:10.1111/j.1460-9568.2006.04853.x

61. Mellott JG, Motts SD, Schofield BR. Multiple origins of cholinergic innervation of the cochlear nucleus. Neuroscience (2011) 180:138-47. doi:10.1016/j. neuroscience.2011.02.010

62. Godfrey DA, Park JL, Dunn JD, Ross CD. Cholinergic neurotransmission in the cochlear nucleus. In: Drescher DG, editor. Auditory Biochemistry. Springfield, IL: Charles C. Thomas (1985). p. 163-83.

63. Chen K, Waller HJ, Godfrey DA. Cholinergic modulation of spontaneous activity in rat dorsal cochlear nucleus. Hear Res (1994) 77:168-76. doi:10.1016/ 0378-5955(94)90264-X

64. Moises HC, Womble MD. Acetylcholine-operated ionic conductances in central neurons. In: Stone TW, editor. CNS Neurotransmitters and Neuromodulators: Acetylcholine. Boca Raton, FL: CRC Press (1995). p. 129-48.

65. Gentschev T, Sotelo C. Degenerative patterns in the ventral cochlear nucleus of the rat after primary deafferentation. An ultra-structural study. Brain Res (1973) 62:37-60. doi:10.1016/0006-8993(73)90618-5

66. Morest DK, Kim J, Bohne BA. Neuronal and transneuronal degeneration of auditory axons in the brainstem after cochlear lesions in the chinchilla: cochleotopic and non-cochleotopic patterns. Hear Res (1997) 103:151-68. doi:10.1016/S0378-5955(96)00172-4

67. Rubel EW, MacDonald GH. Rapid growth of astrocytic processes in n. magnocellularis following cochlea removal. J Comp Neurol (1992) 318:415-25. doi:10.1002/cne.903180406

68. de Waele C, Campos Torres A, Josset P, Vidal PP. Evidence for reactive astrocytes in rat vestibular and cochlear nuclei following unilateral inner ear lesion. Eur J Neurosci (1996) 8:2006-18. doi:10.1111/j.1460-9568.1996.tb01344.x

69. Li H, Godfrey DA, Rubin AM. Astrocyte reaction in the rat vestibular nuclei after unilateral removal of Scarpa's ganglion. Ann Otol Rhinol Laryngol (1999) 108:181-8. doi:10.1177/000348949910800214

70. Powell TP, Erulkar SD. Transneuronal cell degeneration in the auditory relay nuclei of the cat. J Anat (1962) 96:249-68.
71. Morest DK, Bohne BA. Noise-induced degeneration in the brain and representation of inner and outer hair cells. Hear Res (1983) 9:145-51. doi:10.1016/ 0378-5955(83)90024-2

72. Morest DK, Kim J, Potashner SJ, Bohne BA. Long-term degeneration in the cochlear nerve and cochlear nucleus of the adult chinchilla following acoustic overstimulation. Microsc Res Tech (1998) 41:205-16. doi:10.1002/(SICI)10970029(19980501)41:3<205::AID-JEMT4>3.0.CO;2-S

73. Kim JJ, Gross J, Morest DK, Potashner SJ. Quantitative study of degeneration and new growth of axons and synaptic endings in the chinchilla cochlear nucleus after acoustic overstimulation. J Neurosci Res (2004) 77:829-42. doi:10.1002/jnr.20212

74. Feng J, Bendiske J, Morest DK. Degeneration in the ventral cochlear nucleus after severe noise damage in mice. J Neurosci Res (2011) 90:831-41. doi:10. 1002/jnr.22793

75. Takeno S, Wake M, Mount RJ, Harrison RV. Degeneration of spiral ganglion cells in the chinchilla after inner hair cell loss induced by carboplatin. Audiol Neurootol (1998) 3:281-90. doi:10.1159/000013800

76. Ding D-L, Wang J, Salvi R, Henderson D, Hu BH, McFadden SL, et al. Selective loss of inner hair cells and type-I ganglion neurons in carboplatin-treated chinchillas. Mechanisms of damage and protection. Ann N Y Acad Sci (1999) 884:152-70. doi:10.1111/j.1749-6632.1999.tb08640.x

77. Ding D, McFadden SL, Salvi RJ. Calpain immunoreactivity and morphological damage in chinchilla inner ears after carboplatin. J Assoc Res Otolaryngol (2002) 3:68-79. doi:10.1007/s101620020004

78. Potashner SJ, Suneja SK, Benson CG. Regulation of D-aspartate release and uptake in adult brain stem auditory nuclei after unilateral middle ear ossicle removal and cochlear ablation. Exp Neurol (1997) 148:222-35. doi:10.1006/ exnr.1997.6641

79. Muly SM, Gross JS, Potashner SJ. Noise trauma alters D-[3H] aspartate release and AMPA binding in chinchilla cochlear nucleus. J Neurosci Res (2004) 4:585-96. doi:10.1002/jnr.20011

80. Suneja SK, Potashner SJ, Benson CG. AMPA receptor binding in adult guinea pig brain stem auditory nuclei after unilateral cochlear ablation. Exp Neurol (2000) 165:355-69. doi:10.1006/exnr.2000.7471

81. Li H, Godfrey DA, Rubin AM. Quantitative autoradiography of 5- $\left[{ }^{3} \mathrm{H}\right] 6$-cyano7-nitro-quinoxaline-2,3-dione and $(+)-3-\left[{ }^{3} \mathrm{H}\right]$ dizocilpine maleate binding in rat vestibular nuclear complex after unilateral deafferentation, with comparison to cochlear nucleus. Neuroscience (1997) 77:473-84. doi:10.1016/S03064522(96)00468-X

82. Argence M, Saez I, Sassu R, Vassias I, Vidal P, De Waele C. Modulation of inhibitory and excitatory synaptic transmission in rat inferior colliculus after unilateral cochleectomy: an in situ and immunofluorescence study. Neuroscience (2006) 141:1193-207. doi:10.1016/j.neuroscience.2006.04 058

83. Abbott SD, Hughes LF, Bauer CA, Salvi R, Caspary DM. Detection of glutamate decarboxylase isoforms in rat inferior colliculus following acoustic exposure. Neuroscience (1999) 93:1375-81. doi:10.1016/S03064522(99)00300-0

84. Bauer CA, Brozoski TJ, Holder TM, Caspary DM. Effects of chronic salicylate on GABAergic activity in rat inferior colliculus. Hear Res (2000) 147:175-82. doi:10.1016/S0378-5955(00)00130-1

85. Milbrandt JC, Holder TM, Wilson MC, Salvi RJ, Caspary DM. GAD levels and muscimol binding in rat inferior colliculus following acoustic trauma. Hear Res (2000) 147:251-60. doi:10.1016/S0378-5955(00)00135-0

86. Bledsoe SC Jr, Nagase S, Miller JM, Altschuler RA. Deafness-induced plasticity in the mature central auditory system. Neuroreport (1995) 7:225-9. doi:10.1097/00001756-199512290-00054

87. Yang S, Weiner BD, Zhang LS, Cho S, Bao S. Homeostatic plasticity drives tinnitus perception in an animal model. Proc Natl Acad Sci USA (2011) 108:14974-9. doi:10.1073/pnas.1107998108

88. Asako M, Holt AG, Griffith RD, Buras ED, Altschuler RA. Deafness-related decreases in glycine-immunoreactive labeling in the rat cochlear nucleus. $J \mathrm{Neu}$ rosci Res (2005) 81:102-9. doi:10.1002/jnr.20542

89. Buras ED, Holt AG, Griffith RD, Asako M, Altschuler RA. Changes in glycine immunoreactivity in the rat superior olivary complex following deafness. J Comp Neurol (2006) 494:179-89. doi:10.1002/cne.20795

90. Lowry OH, Passonneau JV. A Flexible System of Enzymatic Analysis. New York, NY: Academic Press (1972). 
91. Förster CR, Illing RB. Redistribution of NMDA receptors in the cochlear nucleus following cochleotomy. Neuroreport (1998) 9:3531-5. doi:10.1097/ 00001756-199810260-00036

92. Fyk-Kolodziej B, Shimano T, Gong T-W, Holt AG. Vesicular glutamate transporters: spatio-temporal plasticity following hearing loss. Neuroscience (2011) 178:218-39. doi:10.1016/j.neuroscience.2010.12.059

93. Cohen ES, Brawer JR, Morest DK. Projections of the cochlea to the dorsal cochlear nucleus in the cat. Exp Neurol (1972) 35:470-9. doi:10.1016/00144886(72)90117-3

94. Merchán MA, Collia F-P, Merchán JA, Saldaña E. Distribution of primary afferent fibers in the cochlear nuclei. A silver and horseradish peroxidase (HRP) study. J Anat (1985) 141:121-30.

95. Demediuk P, Daly MP, Faden AI. Effect of impact trauma on neurotransmitter and nonneurotransmitter amino acids in rat spinal cord. J Neurochem (1989) 52:1529-36. doi:10.1111/j.1471-4159.1989.tb09204.x

96. Hua Q, Wang Q, Xiao B. Distribution of gama-aminobutyric acid (GABA)ergic neurons in rats cochlear nuclei after unilateral cochlea ablation. Lin Chuang Er Bi Yan Hou Ke Za Zhi (2005) 19:315-7.

97. Wang Q, Hua Q, Wang S, Xiao B, Liao H. The changes of GABA and GABAergic neurons in inferior colliculus of unilateral cochlear damage rats. Lin Chung Er Bi Yan Hou Tou Jing Wai Ke Za Zhi (2007) 21:321-3.

98. Patel AJ, Hunt A. Concentration of free amino acids in primary cultures of neurones and astrocytes. J Neurochem (1985) 44:1816-21. doi:10.1111/j.14714159.1985.tb07173.x

99. Brozoski TJ, Bauer CA, Caspary DM. Elevated fusiform cell activity in the dorsal cochlear nucleus of chinchillas with psychophysical evidence of tinnitus. J Neurosci (2002) 22:2383-90.

100. Finlayson PG, Kaltenbach JA. Alterations in the spontaneous discharge patterns of single units in the dorsal cochlear nucleus following intense sound exposure. Hear Res (2009) 256:104-17. doi:10.1016/j.heares.2009.07.006

101. Davies WE, Kay IS, Birnso OV. Taurine function in the auditory system. Prog Clin Biol Res (1990) 351:397-405.

102. Brozoski TJ, Caspary DM, Bauer CA, Richardson BD. The effect of supplemental dietary taurine on tinnitus and auditory discrimination in an animal model. Hear Res (2010) 270:71-80. doi:10.1016/j.heares.2010.09.006

103. Chang H, Chen K, Kaltenbach JA, Zhang J, Godfrey DA. Effects of acoustic trauma on dorsal cochlear nucleus neuron activity in slices. Hear Res (2002) 164:59-68. doi:10.1016/S0378-5955(01)00410-5

104. Mugnaini E, Warr WB, Osen KK. Distribution and light microscopic features of granule cells in the cochlear nuclei of cat, rat, and mouse. J Comp Neurol (1980) 191:581-606. doi:10.1002/cne.901910406

105. Chen K, Waller HJ, Godfrey TG, Godfrey DA. Glutamatergic transmission of neuronal responses to carbachol in rat dorsal cochlear nucleus slices. Neuroscience (1999) 90:1043-9. doi:10.1016/S0306-4522(98)00503-X

106. Kaltenbach JA, Godfrey DA. Dorsal cochlear nucleus hyperactivity and tinnitus: are they related? Am J Audiol (2008) 17:S148-61. doi:10.1044/1059-0889(2008/ 08-0004)

107. Manzoor NF, Chen G, Kaltenbach JA. Suppression of noise-induced hyperactivity in the dorsal cochlear nucleus following application of the cholinergic agonist, carbachol. Brain Res (2013) 1523:28-36. doi:10.1016/j.brainres.2013. 05.025
108. Warr WB. Organization of olivocochlear efferent systems in mammals. In: Webster DB, Popper AN, Fay RR, editors. Mammalian Auditory Pathway: Neuroanatomy. New York, NY: Springer-Verlag (1992). p. 410-48.

109. Brown MC, Liberman MC, Benson TE, Ryugo DK. Brainstem branches from olivocochlear axons in cats and rodents. J Comp Neurol (1988) 278:591-603. doi: $10.1002 / \mathrm{cne} .902780410$

110. Cransac H, Cottet-Emard J-M, Hellström S, Peyrin L. Specific sound-induced noradrenergic and serotonergic activation in central auditory structures. Hear Res (1998) 118:151-6. doi:10.1016/S0378-5955(98)00031-8

111. Liu J, Li X, Wang L, Dong Y, Han H, Liu G. Effects of salicylate on serotoninergic activities in rat inferior colliculus and auditory cortex. Hear Res (2003) 175:45-53. doi:10.1016/S0378-5955(02)00708-6

112. Papesh MA, Hurley LM. Plasticity of serotonergic innervation of the inferior colliculus in mice following acoustic trauma. Hear Res (2012) 283:89-97. doi:10.1016/j.heares.2011.11.004

113. Simpson JJ, Davies WE. A review of evidence in support of a role for 5-HT in the perception of tinnitus. Hear Res (2000) 145:1-7. doi:10.1016/S03785955(00)00093-9

114. Oishi N, Kanzaki S, Shinden S, Saito H, Inoue Y, Ogawa K. Effects of selective serotonin reuptake inhibitor on treating tinnitus in patients stratified for presence of depression or anxiety. Audiol Neurootol (2010) 15:187-93. doi:10.1159/000251916

115. Singer W, Zuccotti A, Jaumann M, Lee SC, Panford-Walsh R, Xiong H, et al. Noise-induced inner hair cell ribbon loss disturbs central arc mobilization: a novel molecular paradigm for understanding tinnitus. Mol Neurobiol (2013) 47:261-79. doi:10.1007/s12035-012-8372-8

116. Meleca RJ, Kaltenbach JA, Falzarano PR. Changes in the tonotopic map of the dorsal cochlear nucleus in hamsters with hair cell loss and radial nerve bundle degeneration. Brain Res (1997) 750:201-13. doi:10.1016/S0006-8993(96) 01354-6

Conflict of Interest Statement: The Review Editor James A. Kaltenbach declares that, despite having collaborated with the author Donald A. Godfrey, the review process was handled objectively and no conflict of interest exists. The authors declare that the research was conducted in the absence of any commercial or financial relationships that could be construed as a potential conflict of interest.

Received: 05 August 2014; paper pending published: 08 September 2014; accepted: 20 October 2014; published online: 19 November 2014.

Citation: Lee AC and Godfrey DA (2014) Cochlear damage affects neurotransmitter chemistry in the central auditory system. Front. Neurol. 5:227. doi: 10.3389/fneur.2014.00227

This article was submitted to Neuro-otology, a section of the journal Frontiers in Neurology.

Copyright $(2014$ Lee and Godfrey. This is an open-access article distributed under the terms of the Creative Commons Attribution License (CC BY). The use, distribution or reproduction in other forums is permitted, provided the original author(s) or licensor are credited and that the original publication in this journal is cited, in accordance with accepted academic practice. No use, distribution or reproduction is permitted which does not comply with these terms. 